\title{
Mass-loss and diffusion in subdwarf $B$ stars and hot white dwarfs: do weak winds exist?
}

\author{
K. Unglaub \\ Dr. Remeis-Sternwarte, Astronomisches Institut der Universität Erlangen-Nürnberg, Sternwartstrasse 7, 96049 Bamberg, Germany \\ e-mail: unglaub@sternwarte.uni-erlangen.de
}

Received 5 June 2007 / Accepted 1 April 2008

\begin{abstract}
Context. According to previous investigations, the effect of diffusion in the stellar atmospheres and envelopes of hot white dwarfs and subdwarf B (sdB) stars strongly depends on the presence of weak winds with mass-loss rates $\dot{M}<10^{-11} M_{\odot} / \mathrm{yr}$.

Aims. As in most of these stars with luminosities $L / L_{\odot} \lesssim 100$, no wind signatures have been detected, the mass-loss rates are unknown. In the present paper mass-loss rates are predicted from the original theory of radiatively driven winds.

Methods. The method of solution is modified so that the usual parametrization of the line force multipliers is not necessary. This is important especially for very thin winds. In addition we checked whether a one-component description is justified. As a consequence of various simplifications, the mass-loss rates are expected to be overestimated.

Results. Results are presented for effective temperatures in the range $25000 \mathrm{~K} \leq T_{\text {eff }} \leq 50000 \mathrm{~K}$ and for various metallicities between solar and $Z / Z_{\odot}=0.01$. For (pre-) white dwarfs and sdB stars a stellar mass of $M_{*}=0.5 M_{\odot}$ is assumed. For fixed values of $T_{\text {eff }}, M_{*}$, and $Z$, the results predict decreasing mass-loss rates with increasing surface gravity and an increasing dependence of the mass-loss rates on the metallicity. For white dwarfs with $\log g>7.0$ no wind solution exists even if the metallicity would be solar. Winds with mass-loss rates around $10^{-11}$ to $10^{-10} M_{\odot} / \mathrm{yr}$ are predicted for the most luminous sdB stars with surface gravities of $\log g \lessgtr 5.5$, if the metallicity is not significantly lower than solar. For lower values of $\dot{M}$ metals decouple from hydrogen and helium.

Conclusions. If weak winds with $\dot{M} \lesssim 10^{-12} M_{\odot} / \mathrm{yr}$ exist, the metals cannot be coupled to hydrogen and helium. This should lead to additional changes in the surface composition, which have not yet been taken into account in the diffusion calculations with and without mass-loss. A possible scenario is the existence of pure metallic winds with mass-loss rates of $\dot{M} \lesssim 10^{-16} M_{\odot} / \mathrm{yr}$ and with hydrostatic hydrogen and helium.
\end{abstract}

Key words. hydrodynamics - stars: chemically peculiar - stars: mass-loss - stars: winds, outflows - subdwarfs - white dwarfs

\section{Introduction}

The abundance anomalies in several types of chemically peculiar stars are believed to be at least partially due to diffusion in the stellar atmosphere and envelope. The predictions of diffusion calculations strongly depend on the presence of mass-loss. In several papers (Unglaub \& Bues 1998, 2000, 2001), we investigated the combined effects of gravitational settling, radiative levitation, and weak winds on the chemical composition of hot white dwarfs and subdwarf B (sdB) stars. According to the results, these effects can explain the decreasing abundances of helium and metals during the cooling process of white dwarfs on the upper cooling sequence (with effective temperatures $T_{\text {eff }} \gtrsim$ $50000 \mathrm{~K}$ ). The mass-loss rates were estimated either from scaling laws or were a free parameter. The winds were assumed to be "chemically homogeneous". If $\dot{M}_{1}$ is the mass-loss rate of an element and $\zeta_{1}$ its mass fraction in the photosphere, then this assumption states that $\dot{M}_{1}=\zeta_{1} \dot{M}$, where $\dot{M}$ is the total mass-loss rate. Such a wind prevents (if $\dot{M} \gtrsim 10^{-11} M_{\odot} / \mathrm{yr}$ ) or retards (if $\left.\dot{M}<10^{-11} M_{\odot} / \mathrm{yr}\right)$ gravitational settling. The present paper investigates whether the assumed mass-loss rates are consistent with the predictions of the theory of radiation-driven winds and whether these winds may be chemically homogeneous.

In thin winds the one-component description is questionable, because the momentum redistribution via Coulomb collisions between metals (which are preferably accelerated due to the radiative flux) and hydrogen and helium may not be effective enough (e.g. Owocki \& Puls 2002; Krtička et al. 2003). This may lead to selective winds in which the metals are expelled from the star, whereas hydrogen and helium are left behind (Babel 1995, 1996). In contrast to chemically homogeneous winds, selective winds should directly change the surface composition.

In the absence of any mass-loss and other disturbing processes (e.g. convective mixing), an equilibrium between gravitational settling and radiative levitation should be expected. Because of saturation effects the radiative force on an element in the stellar atmosphere and envelope depends on its abundance. The radiative force decreases with increasing abundance, so an "equilibrium abundance" can be found, for which the radiative force balances the effect of gravitational settling. This theory is described in Chayer et al. (1995a,b) and references therein. Dreizler \& Wolff (1999) and Schuh et al. (2002) have incorporated the diffusion theory into their NLTE stellar atmosphere code, so synthetic spectra have been calculated for an abundance stratification, which is given from the equilibrium condition between gravitational settling and radiative levitation. However, for hot white dwarfs the quantitative agreement with observational results is not satisfactory in many cases. The presence of weak winds may be a possible reason for these discrepancies.

Winds have been detected in pre-white dwarfs with effective temperatures in the range $30000 \mathrm{~K} \lesssim T_{\text {eff }} \lesssim 150000 \mathrm{~K}$ and surface gravities $3.5 \lessgtr \log g \lessgtr 6.0$ (cgs units). These objects are in a post-asymptotic giant branch stage of evolution, and they evolve with approximately constant luminosities close 
to $10^{4} L_{\odot}$ to higher effective temperatures. As derived from observations and theoretical calculations (see e.g. Kudritzki et al. 2006; Pauldrach et al. 2004; Tinkler \& Lamers 2002; Herald et al. 2005; Koesterke et al. 1998; Koesterke \& Werner 1998, and the review of Kudritzki \& Puls 2000), the mass-loss rates are between $10^{-6}$ and $10^{-9} M_{\odot} / \mathrm{yr}$. The mass-loss rates of white dwarfs on the cooling sequence (if winds exist at all) are unknown.

The sdB stars can be identified with models of the extreme horizontal branch (EHB) stars with masses of $M_{*} \approx$ $0.5 M_{\odot}$ (Heber 1986; Saffer et al. 1994), effective temperatures of $20000 \mathrm{~K} \leq T_{\text {eff }} \leq 40000 \mathrm{~K}$, and surface gravities of $5.0 \lessgtr \log g \lesssim 6.0$. Helium is usually deficient with number ratios of $10^{-4} \lesssim \mathrm{He} / \mathrm{H} \lesssim 0.1$ (e.g. Edelmann et al. 2003; Lisker et al. 2005). However, these helium abundances are by at least one order of magnitude more than would be expected from the equilibrium condition between gravitational settling and radiative levitation (Michaud et al. 1989). A possible explanation for this discrepancy is the presence of weak winds that retard gravitational settling (Fontaine \& Chayer 1997; Unglaub \& Bues 1998). For mass-loss rates $\dot{M} \approx 10^{-13} M_{\odot} /$ yr, within the lifetimes of $\mathrm{sdB}$ stars near the EHB $\left(\approx 10^{8} \mathrm{yr}\right)$, the helium abundances would gradually decrease from the solar value to $\mathrm{He} / \mathrm{H} \approx 10^{-4}$.

The helium deficiencies in sdB stars are accompanied by anomalies in the abundances of heavy elements. Some of the most recent results of spectral analyses are from Geier et al. (2008); O'Toole \& Heber (2006); Edelmann et al. (2006); Blanchette et al. (2006), and Chayer et al. (2006). The hotter sdBs with $T_{\text {eff }}>30000 \mathrm{~K}$ show strong deficiencies in many cases especially of light metals like $\mathrm{Al}, \mathrm{Mg}, \mathrm{O}$, and $\mathrm{Si}$ by more than a factor of 100 in comparison to the solar abundances, whereas enrichments of elements heavier than iron by a factor near 100 are not unusual. The abundances of iron and nitrogen are usually close to the solar value. If the scenario with diffusion and chemically homogeneous winds were the correct explanation of these compositions, it should be possible to find a mass-loss rate for which the anomalies of all elements can be explained simultaneously. For the case with solar initial abundance, the calculations of Unglaub \& Bues (2001) show that, in the case of chemically homogeneous winds, helium should always be more deficient than at least the elements $\mathrm{C}, \mathrm{N}$, and $\mathrm{O}$, which have been taken into account. No mass-loss rate exists that leads to deficiencies in $\mathrm{C}$ and $\mathrm{O}$ by more than a factor of 100 , whereas helium is only deficient by a factor of ten. This, however, is not an unusual composition in sdB stars (e.g. Heber et al. 2000).

Pulsating and non-pulsating sdB stars with similar stellar parameters coexist in the $T_{\text {eff }}-\log g$ diagram (Charpinet et al. 2006). The proposed pulsation mechanism is associated with a local enhancement of iron (or other iron group elements) in a mass depth of about $10^{-7} M_{*}$, which is indeed expected from the equilibrium condition between gravitational settling and radiative levitation (Charpinet et al. 1997; Fontaine et al. 2003). However, according to the calculations of Chayer et al. (2004) and Fontaine et al. (2006), a weak wind with only $\dot{M}=6 \times 10^{-15} M_{\odot} /$ yr would be sufficient to destroy the iron reservoir within a timescale of $10^{7} \mathrm{yr}$. For higher mass-loss rates, this should happen in even shorter time scales. Thus for $\dot{M} \approx 10^{-13} M_{\odot} / \mathrm{yr}$, which would be required to explain the helium abundances, this pulsation mechanism would become questionable.

Mass-loss has been detected in sdO stars that are more luminous than sdBs (Hamann et al. 1981; Rauch 1993). For sdB stars, up to now there has been no observational proof for the existence of winds. From a quantitative analysis of the $\mathrm{H} \alpha$ line profiles of $40 \mathrm{sdB}$ stars (Maxted et al. 2001), a comparison of synthetic NLTE $\mathrm{H} \alpha$ line profiles from static model atmospheres with the observations have revealed perfect matches for almost all stars. Only in the four most luminous sdBs have anomalous $\mathrm{H} \alpha$ lines with a small emission at the line centre been detected, which are possibly signatures of weak winds (Heber et al. 2003). For the case with $T_{\text {eff }}=36000 \mathrm{~K}, \log g=5.5$, and $\log L / L_{\odot}=1.51$, Vink (2004) found a similar behaviour of $\mathrm{H} \alpha$ from a spectral analysis of $\mathrm{H} \alpha$ with his wind code, if the existence of a weak wind with $\dot{M} \approx 10^{-11} M_{\odot} /$ yr is assumed.

For several effective temperatures in the range $25000 \mathrm{~K} \leq$ $T_{\text {eff }} \leq 50000 \mathrm{~K}$ and for various metallicities, mass-loss rates will be predicted according to the theory of radiation-driven winds from Castor et al. (1975, henceforth CAK). As the usual scaling laws may be unreliable for thin winds, the method of solution has been changed slightly (see Sect. 2), so it is taken into account that the radiative force is limited for small wind optical depth parameters and tends to some maximum value. To simplify the numerical method, later improvements in the CAK theory are neglected. As discussed in Sect. 6, it should be possible to derive at least an upper limit for the mass-loss rates. For each wind model, we checked whether a one-component description may be justified (see Sect. 3). The results for $T_{\text {eff }}=40000$ and $50000 \mathrm{~K}$ and several metallicities, which have been obtained with line force multipliers according to Kudritzki (2002), are presented in Sect. 4. The results for $T_{\text {eff }}=25000,30000$, and $35000 \mathrm{~K}$ (Sect. 5) were obtained with force multipliers according to our own calculations (see Sect. 2.4.2). The predicted mass-loss rates are compared with the results of Vink \& Cassisi (2002).

In Sects. 6.3 and 6.4, we discuss how the abundance anomalies in sdB stars could be explained. The arguments are also relevant for hot white dwarfs and chemically peculiar main sequence stars, which are reviewed by Smith (1996). A subgroup are the HgMn stars. Because they are characterised by very low rotational velocities and weak or non-detectable magnetic fields, they are one of the best natural laboratories for studying the competing processes of gravitational settling and radiative diffusion (Vauclair \& Vauclair 1982). The HgMn stars are characterised by enhancements of heavy metals (e.g. $\mathrm{Hg}, \mathrm{Mn}, \mathrm{Pt}, \mathrm{Sr}$, $\mathrm{Ga}$ ), deficiencies of some light elements (e.g. He, Al, N), and isotopic anomalies of metals. Some of the most recent papers about these stars are from Zavala et al. (2007) and Adelman et al. (2006). Similar to sdB stars, weak winds with mass-loss rates of $10^{-14}$ to $10^{-12} M_{\odot} /$ yr could lead to abundance anomalies that are different from the ones obtained from the equilibrium condition between gravitational settling and radiative levitation (Landstreet et al. 1998). However, for the typical stellar parameters $10500 \mathrm{~K} \leq T_{\text {eff }} \leq 16000 \mathrm{~K}$ and $\log g \approx 4.0$, these winds can hardly be chemically homogeneous, because the radiative force is too low (Babel 1996).

In chemically peculiar main sequence stars, the abundance anomalies depend on stellar rotation (see e.g. Vauclair 2003), on the presence of magnetic fields (Turcotte 2003) and of convection zones. In hydrogen-rich hot white dwarfs and sdB stars, these effects do not seem to be the most important ones. In general both are slow rotators (Karl et al. 2005; Koester et al. 1998; Heber et al. 1997; Heber \& Edelmann 2004). O'Toole (2005) finds no correlation between magnetic field strengths in $\mathrm{sdB}$ stars and abundance anomalies. In some (pre-) white dwarfs, magnetic fields have been detected (Jordan et al. 2005, 2007, and references therein). However, diffusion is effective in all white dwarfs and not restricted to a subgroup of them. A thin 
superficial convection zone with a mass depth of about $10^{-12} M_{*}$ may be present in sdB stars, but only for helium abundances $\mathrm{He} / \mathrm{H} \gtrsim 0.01$ by number (Groth et al. 1985).

\section{Calculation of mass-loss rates}

CAK introduced the dimensionless optical depth parameter

$t=\sigma_{\mathrm{e}} v_{\mathrm{th}} \rho\left(\frac{\mathrm{d} v}{\mathrm{~d} r}\right)^{-1}$,

where $\rho$ is the density and $\mathrm{d} v / \mathrm{d} r$ is the velocity gradient. Here, $\sigma_{\mathrm{e}}$ is the electron scattering opacity with

$\sigma_{\mathrm{e}}=\frac{n_{\mathrm{e}}}{\rho} \sigma_{\mathrm{T}}$

where $\sigma_{\mathrm{T}}=6.6524 \times 10^{-25} \mathrm{~cm}^{2}$ is the Thomson cross-section for electrons and $n_{\mathrm{e}}$ the electron number density. The ratio $n_{\mathrm{e}} / \rho$ depends on the composition and on the degree of ionization. Both are assumed to be constant throughout the wind. In addition, the wind is assumed to be isothermal with $T=T_{\text {eff }}$. Thus the mean thermal velocity $v_{\text {th }}$ of hydrogen is

$v_{\text {th }}=\sqrt{\frac{2 k_{\mathrm{B}} T_{\mathrm{eff}}}{m_{\mathrm{H}}}}$

where $k_{\mathrm{B}}$ is the Boltzmann constant and $m_{\mathrm{H}}$ the atomic mass of hydrogen. The line radiative acceleration can be written in terms of the line force multiplier $M(t)$ and the luminosity $L$ of the star

$g_{\mathrm{rad}}=\frac{1}{c} \sigma_{\mathrm{e}} \frac{L}{4 \pi r^{2}} M(t)$.

In the original CAK theory, the force multiplier is parametrized according to $M(t)=k t^{-\alpha}$. With constant force multiplier parameters $k$ and $\alpha$ and if the radiative acceleration due to electron scattering is neglected, it follows that $\dot{M} \sim L^{\frac{1}{\alpha}}$ (see e.g. Puls et al. 2000). Usually $\alpha$ is between about 0.5 and 0.7 . However, the results of such scaling laws are questionable for the case of thin winds. This is because, according to the parametrized form, the force multiplier and thus the radiative force may become arbitrarily strong if the wind optical depth parameter is small enough. As a consequence, even for such compact stars as white dwarfs, for which in fact no wind solution exists, the scaling laws derived for more luminous stars predict small, but non-zero mass-loss rates.

For $t \rightarrow 0$ the $\log M(t)-\log t$ is not a straight line as would follow from the parametrized form, but the relation flattens and the slope $-\alpha$ of this relation approaches zero (see Sects. 2.4 and 5.1). For given excitation and ionization equilibrium, the force multiplier smoothly tends to a maximum value $M_{\max }$. To take this into account, Kudritzki (2002) developed a method with variable force multiplier parameters to calculate wind models for extremely metal-deficient hot stars. The methods used in the present paper as described in Sects. 2.1 and 2.2 allow the calculation of the mass-loss rate without parametrization of the force multiplier. However, in contrast to Kudritzki's method, later improvements in the CAK theory like the finite disk correction factor (Pauldrach et al. 1986; Friend \& Abbott 1986) and changes in ionization in the wind are neglected.

For fixed excitation and ionization equilibrium, the force multiplier depends on the wind optical depth parameter alone (see Sect. 2.4.2). With the equation of continuity

$\dot{M}=4 \pi r^{2} \rho v$ and the definition

$D=r^{2} v \frac{\mathrm{d} v}{\mathrm{~d} r}$

the wind optical depth parameter can be written as

$t=\sigma_{\mathrm{e}} v_{\mathrm{th}} \frac{\dot{M}}{4 \pi D}$

so $t$ and thus the force multiplier are a function of $\dot{M}$ and $D$. Then, as described below, the solution to the momentum equation is straightforward. The inclusion of the improvements in the CAK theory would lead to additional dependencies, because the finite disk correction factor, as well as the ionization equilibrium, change with radius. This may complicate the method of solution. Moreover, in weak winds these effect are probably less important than the effect of the shadowing of the flux by the photospheric lines discussed by Babel (1996), which is not taken into account in the original CAK theory and thus in the present calculations. The consequences of the various simplifications and their relative importance will be discussed in Sect. 6.

The momentum equation for a stationary wind is

$v \frac{\mathrm{d} v}{\mathrm{~d} r}=-\frac{1}{\rho} \frac{\mathrm{d} p}{\mathrm{~d} r}-\frac{G M_{*}}{r^{2}}\left(1-\Gamma_{\mathrm{e}}\right)+g_{\mathrm{rad}}$

where $\mathrm{d} p / \mathrm{d} r$ is the gradient of the gas pressure, $G$ the gravitational constant, and $\Gamma_{\mathrm{e}}$ is the Eddington factor:

$\Gamma_{\mathrm{e}}=\frac{L \sigma_{\mathrm{e}}}{4 \pi c G M_{*}}$

For sdB stars, $\Gamma_{\mathrm{e}}$ is only about 0.01 . The solution to the momentum equation is especially simple, if the contribution of the gas pressure is completely (see Sect. 2.1) or partially (Sect. 2.2) neglected.

\subsection{Momentum equation without gas pressure}

If the contribution of the gas pressure to the momentum equation is neglected and if the force multiplier is given in parametrized form with constant parameters, then analytical formulae for the mass-loss rate and terminal velocity can be derived (see Sect. 8.7.1 of Lamers \& Cassinelli 1999). The method is similar for non-parametrized force multipliers; but then the equations must be solved numerically. If in Eq. (8) $\mathrm{d} p / \mathrm{d} r$ is neglected, Eq. (4) for $g_{\text {rad }}$ inserted, and the resulting equation multiplied with $r^{2}$, then the momentum equation with the definition of $D$ can be written as

$H \equiv D+G M_{*}\left(1-\Gamma_{\mathrm{e}}\right)-\frac{L \sigma_{\mathrm{e}}}{4 \pi c} M(t)=0$.

With the present assumptions, this equation only depends on $\dot{M}$ and $D$. All other quantities are fixed. For a given mass-loss rate, it has either two solutions, one or no solution for $D$. The massloss rate according to the CAK theory corresponds to the case with one solution. Then the critical point of CAK degenerates and every point in the flow becomes critical. This solution fulfils the critical point condition $\mathrm{d} H / \mathrm{d}(\mathrm{d} v / \mathrm{d} r)=0$, which is equivalent to

$\frac{\mathrm{d} H}{\mathrm{~d} D}=0$.

With Eq. (10), it follows that

$1-\frac{L \sigma_{\mathrm{e}}}{4 \pi c} \frac{\mathrm{d} M(t)}{\mathrm{d} t} \frac{\mathrm{d} t}{\mathrm{~d} D}=0$ 
Equations (10) and (12) are solved numerically for $\dot{M}$ and for the critical value $D_{\mathrm{c}}$. From insertion of $\dot{M}$ and $D_{\mathrm{c}}$ into Eq. (7) the critical optical depth parameter $t_{\mathrm{c}}$ follows, which for completely neglected gas pressure is constant throughout the flow. The solution $v(r)$ is obtained by quadrature of Eq. (6) with $D=D_{\text {c }}$, so that

$v(r)=\left(v_{0}^{2}+\frac{2 D_{\mathrm{c}}}{R_{*}}\left(1-\frac{R_{*}}{r}\right)\right)^{\frac{1}{2}}$

where $v_{0}$ is the velocity at $r=R_{*}$. For $r \rightarrow \infty$ and with $v_{0} \ll v_{\infty}$ the terminal velocity is

$v_{\infty}=\sqrt{\frac{2 D_{\mathrm{c}}}{R_{*}}}$.

This gives the usual $\beta$ - type velocity law with $\beta=\frac{1}{2}$.

\subsection{The shooting method}

To neglect the gas pressure completely is only justified in the supersonic region, where the wind essentially is driven by the radiative force. If one is interested in the solution $v(r)$ of the momentum equation in the region around the sonic point and in the subsonic region, the gas pressure must at least be partially taken into account. With the equation of continuity and the equation of state for a perfect gas, the gradient of the gas pressure can be written as

$\frac{\mathrm{d} p}{\mathrm{~d} r}=-\rho\left(\frac{a^{2}}{v} \frac{\mathrm{d} v}{\mathrm{~d} r}+\frac{2 a^{2}}{r}\right)$.

Here $a$ is the isothermal sound speed. With $T=T_{\text {eff }}$, it is

$a=\sqrt{\frac{k_{\mathrm{B}} T_{\mathrm{eff}}}{\mu}}$

where $\mu$ is the mean particle mass. The first term in brackets on the righthand side of Eq. (15) corresponds to an acceleration caused by the gradient of the gas pressure in planar geometry. This is the contribution to the momentum equation, which is important in the subsonic region near the photosphere of the star. The term $2 a^{2} / r$ is the acceleration due to the area expansion.

In winds with temperatures $T \approx T_{\text {eff }}$, the gravitational acceleration $g(r)$ exceeds this curvature term by more than a factor of 100 near the photosphere. As, however, $g(r)$ decreases proportionaly to $r^{-2}$, whereas the curvature term decreases only with $r^{-1}$, a radius exists at which both accelerations are equal. This is denoted as the Parker point (Lamers \& Cassinelli 1999) and is located far outside the star at distances of some hundreds of stellar radii. In a wide region between the sonic point and the Parker point, the contribution of the gas pressure to the momentum equation is small. As we intend to solve the momentum equation by outward integration from the photosphere, at least the first part of $\mathrm{d} p / \mathrm{d} r$, which is important in the subsonic region, must be taken into account. The curvature term, however, will be neglected. Then with Eqs. (8) and (15) it follows that

$v \frac{\mathrm{d} v}{\mathrm{~d} r}\left(1-\frac{a^{2}}{v^{2}}\right)=-\frac{G M_{*}}{r^{2}}\left(1-\Gamma_{\mathrm{e}}\right)+g_{\mathrm{rad}}$

In the complete momentum equation the term $2 a^{2} / r$ should appear on the righthand side. The omission of this term simplifies the solution topology and the numerical method (see Sect. 2.3). As the density in the photosphere is known from the hydrostatic equation, the velocity $v_{0}$ at the inner boundary (at $r=R_{*}$, which is assumed at a Rosseland mean optical depth $\bar{\tau}=2 / 3$ ) can be obtained for a trial value of $\dot{M}$ from the equation of continuity. With this initial value, Eq. (17) is integrated outwards. The highest mass-loss rate, for which a solution can be extended from the photosphere to infinity, is the one according to the CAK theory. For all cases discussed in the present paper, the mass-loss rates have been calculated from both methods described in Sects. 2.1 and 2.2. The results are very similar, because the differences do not exceed numerical uncertainties.

\subsection{Example: 05 main sequence star}

To illustrate the methods described in the previous sections, we now calculate a wind model for an O5 main sequence star using the same stellar parameters as CAK:

$T_{\text {eff }}=49290 \mathrm{~K}, \quad \log g=3.94, \quad M_{*}=60 M_{\odot}$,

and $L=9.66 \times 10^{5} L_{\odot}, \Gamma_{\mathrm{e}}=0.4, \sigma_{\mathrm{e}}=0.325 \mathrm{~cm}^{2} \mathrm{~g}^{-1}$. As in $\mathrm{CAK}$ for the force multiplier it is assumed that

$M(t)=\frac{1}{30}\left(\frac{1}{\sqrt{12}} t\right)^{-0.7}$

The factor $1 / \sqrt{12}$ appears because CAK used the optical depth parameter for a carbon ion, so that their $v_{\text {th }}$ is the thermal velocity of carbon. Although $M(t)$ is given in parametrized form, we do not make use of the parametrization during the calculations.

Solutions for $\dot{M}=10^{-4}$ and $10^{-5} M_{\odot} / \mathrm{yr}$ fail at velocities somewhat higher than the sound speed. Thus these mass-loss rates are too high. The highest mass-loss rate for which the solution can be extended outwards to infinity and the corresponding terminal velocity are

$\dot{M}=6.4 \times 10^{-6} \frac{M_{\odot}}{\mathrm{yr}} \quad v_{\infty}=1527 \mathrm{~km} \mathrm{~s}^{-1}$

For comparison, CAK obtained $\dot{M}=6.6 \times 10^{-6} M_{\odot} / \mathrm{yr}$ and $v_{\infty}=1515 \mathrm{~km} \mathrm{~s}^{-1}$. If the finite disk correction factor were taken into account and all other assumptions are unchanged, then Pauldrach et al. (1986) obtain $\dot{M}=3.5 \times 10^{-6} M_{\odot} / \mathrm{yr}$ and $v_{\infty}=5123 \mathrm{~km} \mathrm{~s}^{-1}$. After multiplication of Eq. (17) with $r^{2}$, with Eq. (4) for $g_{\mathrm{rad}}$, it follows that with the present assumptions Eq. (17) depends on $\dot{M}, D$, and $v$. In the limit $v \gg a$, it is identical with the momentum equation with completely neglected gas pressure. Thus for high velocities the solutions of Eqs. (10) and (17) approach each other, whereas in the inner regions, where the gas pressure is essential, the differences are great, as can be seen from comparing the solid and dashed lines in Fig. 1a. In addition to the critical solution, shallow solutions also exist. They are characterised by lower mass loss rates, e.g. $\dot{M}=10^{-6}$ or $10^{-7} M_{\odot} / \mathrm{yr}$ and terminal velocities below the CAK value.

In Fig. 1b the solutions $D$ of Eq. (17) are shown as a function of the velocity. In the subsonic region one solution for $D$ (and thus the velocity gradient) exists. In the supersonic region, however, two solutions exist for each mass-loss rate: a shallow solution with a low value of $D$ and a steep one with a high value of $D$. For mass-loss rates higher than the critical value, the shallow and steep solution meet, so that for velocities somewhat higher than $a$, no solution of the momentum equation exists any more. The critical value of $\dot{M}$ is the highest mass-loss rate for which both the shallow and the steep solution can be extended outwards. These solutions approach each other and for $v \gg a$ 

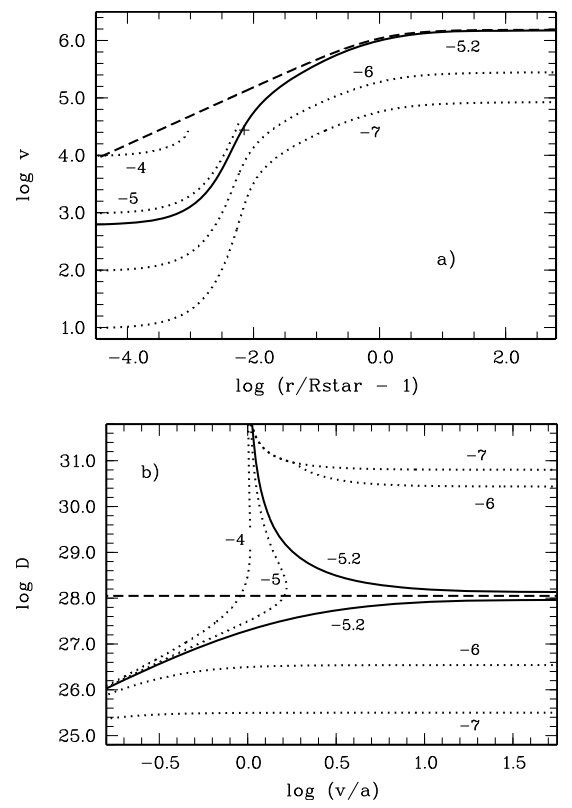

Fig. 1. Results for $T_{\text {eff }}=49290 \mathrm{~K}, \log g=3.94, M_{*}=60 M_{\odot}$. a) Solutions $v(r)$ (in $\mathrm{m} \mathrm{s}^{-1}$ ) obtained with the shooting method for $\dot{M}=6.4 \times 10^{-6} M_{\odot} / \mathrm{yr}$ (solid line; the cross indicates the location of the sonic point), $\dot{M}=10^{-4}, 10^{-5}, 10^{-6}$ and $10^{-7} M_{\odot} / \mathrm{yr}$ (dotted lines). The dashed line is the critical solution of the momentum equation with completely neglected gas pressure. b) Solutions $D\left(\right.$ in $\left.\mathrm{cm}^{3} \mathrm{~s}^{-2}\right)$ of the momentum Eq. (17) as a function of the velocity for the mass-loss rates as in Fig. 1a. The horizontal dashed lines represents the critical value $D_{\text {c }}$ obtained from the solution of the momentum equation with completely neglected gas pressure. In both figures the curves are labelled with $\log \dot{M}$.

they converge to the critical solution of the momentum equation with completely neglected gas pressure (in this case $D$ is constant). For subcritical mass-loss rates, the shallow and the corresponding steep solution converge to different values of $D$ for $v \gg a$.

The solution topology of the complete momentum equation with the curvature term taken into account is more complicated (Bjorkman 1995). It has an X-type topology in $D$ and several critical points exist. Then the shallow solutions meet at the Parker point and cannot be continously (in $D$ ) extended outwards. For this reason they are ruled out by CAK. Then for only one mass-loss rate a smooth and continuous solution exists, which reaches from the photosphere to infinity. To find this solution, it is necessary to switch from a shallow to a steep solution at the CAK critical point. As in the present case with neglected curvature term shallow solutions can also be extented from the photosphere to infinity, only an upper limit can be set for the mass-loss rate from the time-independent equations. From timedependent calculations, however, Feldmeier \& Shlosman (2002) suggest that shallow solutions evolve in time towards higher velocities and mass-loss rates. Thus their physical relevance is questionable.

As the steep solutions do not exist in the subsonic region, the outward integration of the momentum equation from the photosphere must start on a shallow solution. As for the critical mass-loss rate, the shallow and steep solution are identical in the limit $v \gg a$ (which would not be the case if the curvature term were taken into account), the critical solution can be found without switching from the shallow to the steep solution. This simplifies the numerical method, especially in the case of weak winds, in which the wind optical parameter is in a range where the $\log M(t)-\log t$ relation is curved. Due to the X-type solution topology of the complete momentum equation, the integration should start at the critical point in this case. This is the method used by CAK. To set the initial conditions at the CAK critical point, the knowledge of the slope of the $\log M(t)-\log t$ relation (which corresponds to the force multiplier parameter $\alpha$ ) is required. Therefore this method leads to complications, if this relation is curved and thus $\alpha$ is not a constant.

As can be seen from Fig. 1b, a solution with a subcritical mass-loss rate and a terminal velocity higher than the CAK value would require a jump in $D$ from a shallow onto a steep solution somewhere in the supersonic region (higher values of $D$ lead to higher terminal velocities as can be seen from quadrature of Eq. (6)). To see if a discontinuity in $D$ may be physically reasonable, the time-dependent equations should be considered. As, however, the CAK theory is based on the use of the Sobolev approximation for calculating the radiative force, the mathematical nature of the momentum equation would change if the Sobolev approximation were dropped (Lucy 2007a,b). As the Sobolev approximation is questionable at least in the subsonic region, it cannot be excluded that smooth and continuous solutions with subcritical mass-loss rates and high terminal velocities indeed exist in this case.

\subsection{The force multiplier}

The results for $T_{\text {eff }}=40000 \mathrm{~K}$ and $50000 \mathrm{~K}$, which will be presented in Sect. 4, were obtained with force multipliers according to Kudritzki (2002). To avoid the methods of solution described in Sects. 2.1 and 2.2 being complicated by changing ionization and excitation equilibrium, the dependence of Kudritzki's force multipliers on the degree of ionization is only approximately taken into account. How this is done, is described in Sect. 2.4.1.

The results for $T_{\text {eff }}=25000,30000$, and $35000 \mathrm{~K}$, which will be presented in Sect. 5, have been obtained with force multipliers from own calculations. These calculations and the various assumptions are described in Sect. 2.4.2.

\subsubsection{Force multipliers according to Kudritzki (2002)}

For $T_{\text {eff }}=40000,50000$, and $60000 \mathrm{~K}$, Kudritzki (2002) calculated force multipliers with a line list of $2.5 \times 10^{6}$ lines of 150 ionic species and with approximate non-LTE occupation numbers as a function of the wind optical depth parameter and of $n_{\mathrm{e}} / W$, where $n_{\mathrm{e}}$ is the electron density and $W$ the geometrical dilution factor $(W \approx 0.5$ near the photosphere).

In the present calculations $n_{\mathrm{e}} / W$ is assumed to be fixed throughout the wind. The dependence on the degree of ionization is taken into account with the following iteration procedure. A first wind model is calculated with an input value (e.g. $n_{\mathrm{e}} / W=10^{11} \mathrm{~cm}^{-3}$ ). From this model we obtain the mean value of $n_{\mathrm{e}} / W$ at the sonic point and at a radius $r=2 R_{*}$. The iteration is complete, if this output value is consistent with the input value within an accuracy of about $10 \%$. Otherwise the iteration is repeated with an improved input value to ensure that the value of $n_{\mathrm{e}} / W$ used in the calculation of the force multipliers has a similar order of magnitude to the value of the corresponding wind model near the sonic point and the CAK critical point (which according to the original theory with the complete momentum equation is near $r=1.5 R_{*}$ ).

In Fig. 2 for $T_{\text {eff }}=40000$ and $50000 \mathrm{~K}$ and for $Z / Z_{\odot}=1$, the mass-loss rates calculated with this iteration procedure are compared to the results obtained with the assumption that it is always 

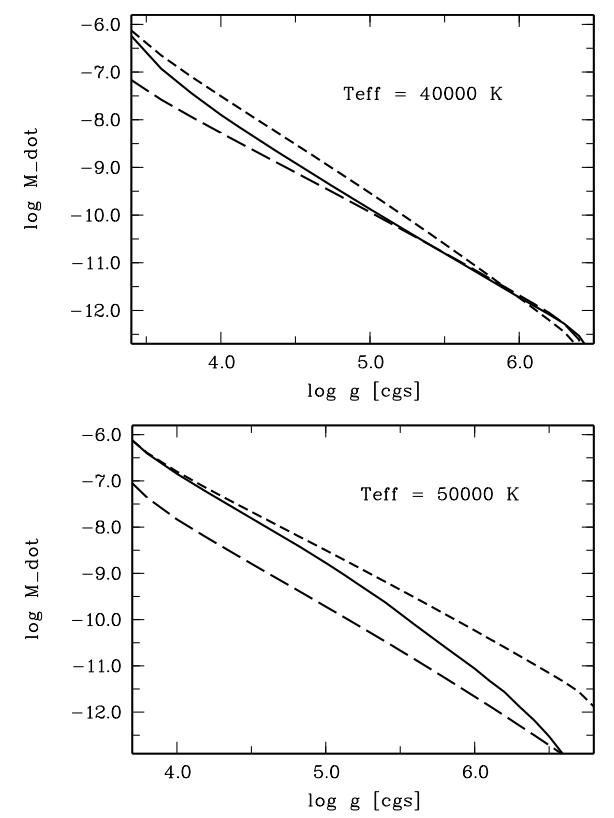

Fig. 2. Mass-loss rates (in $M_{\odot} / \mathrm{yr}$ ) as a function of surface gravity for $T_{\text {eff }}=40000 \mathrm{~K}, 50000 \mathrm{~K}$, and $Z / Z_{\odot}=1$ calculated with force multipliers according to Kudritzki (2002). The solid lines represent the results obtained with the iteration procedure for $n_{\mathrm{e}} / W$ as described in the text. The short-dashed and long-dashed lines represent the results for $n_{\mathrm{e}} / W=10^{14}$ and $10^{10} \mathrm{~cm}^{-3}$, respectively.

$n_{\mathrm{e}} / W=10^{14} \mathrm{~cm}^{-3}$ or $10^{10} \mathrm{~cm}^{-3}$, respectively, regardless how high the wind density is. It can be seen that, for $T_{\text {eff }}=40000 \mathrm{~K}$ and $\log g \gtrsim 5.0$, the results are approximately the same in each case with deviations less than a factor of two. This is because the dependence of the force multiplier on $n_{\mathrm{e}} / W$ is weak for small wind optical depth parameters, which are expected in thin winds (see Fig. 3). The situation is different for $T_{\text {eff }}=50000 \mathrm{~K}$. Here the mass-loss rates derived for the two values of $n_{\mathrm{e}} / W$ may differ by almost two orders of magnitude. The values obtained with the iteration procedure are in between these results (the finally adopted values of $n_{\mathrm{e}} / \mathrm{W}$ can be read off from Fig. 5 in Sect. 4).

For $T_{\text {eff }}=60000 \mathrm{~K}$, the dependence of the mass-loss rates on the degree of ionization is even stronger; e.g., for log $g=6.0$ it follows that $\log \dot{M}=-11.4$ with $n_{\mathrm{e}} / W=10^{10} \mathrm{~cm}^{-3}$ and $\log \dot{M}=-7.6$ with $n_{\mathrm{e}} / W=10^{14} \mathrm{~cm}^{-3}$. Thus the predicted value of $\dot{M}$ depends almost linearily on $n_{\mathrm{e}} / W$. Then the iteration procedure for $n_{\mathrm{e}} / W$ does not converge. For this reason the case $T_{\text {eff }}=60000 \mathrm{~K}$ is not considered in the present paper.

The force multipliers according to Kudritzki's Eq. (25) have been not allowed to exceed a value of $M_{\max }=2000 \mathrm{Z} / \mathrm{Z}_{\odot}+$ $M_{\mathrm{H}, \mathrm{He}}$, according to his Eq. (12). The contribution $M_{\mathrm{H}, \mathrm{He}}$ of hydrogen and helium to $M_{\max }$ was estimated from own calculations as described below. The adopted values are $M_{\mathrm{H}, \mathrm{He}}=263$ for $T_{\text {eff }}=40000 \mathrm{~K}$ and $M_{\mathrm{H}, \mathrm{He}}=93$ for $T_{\text {eff }}=50000 \mathrm{~K}$. At least for $T_{\mathrm{eff}}=40000 \mathrm{~K}, Z / Z_{\odot}=1$ this restriction is in good agreement with the value $M_{\max }=2340$ obtained from own calculations (see Fig. 3). It only affects the results for extremely weak winds with wind optical depth parameters $\log t \lesssim-7.0$. In these cases decoupling of the ions from hydrogen and helium is expected, so the corresponding wind models are of questionable physical relevance. Thus this restriction does not change the conclusion of the present paper.

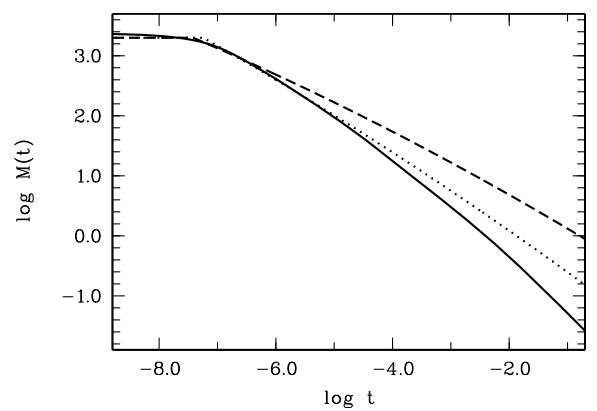

Fig. 3. Line force multipliers for $T_{\text {eff }}=40000 \mathrm{~K}, Z / Z_{\odot}=1$ as a function of the wind optical depth parameter according to own calculations (solid line) and according to Eq. (25) of Kudritzki (2002) for $n_{\mathrm{e}} / W=10^{14}$ (dashed line) and $10^{10} \mathrm{~cm}^{-3}$ (dotted line). Kudritzki's values have been not allowed to exceed $\log M_{\max }=3.37$.

\subsubsection{Force multipliers from own calculations}

The results presented in Sect. 5 are obtained with force multipliers from own calculations. The elements $\mathrm{H}, \mathrm{He}, \mathrm{C}, \mathrm{N}$, and $\mathrm{O}$ are taken into account with a line list that is essentially similar to the one used in the diffusion calculations of Vauclair et al. (1979) and later in our diffusion calculations (Unglaub \& Bues 1996). It consists of about 150 preferably strong lines with atomic data from Wiese et al. (1966). Similar to the diffusion calculations, multiplets, or doublets are lumped together into one line (note, however, that this may underestimate the radiative force, if not all lines are optically thin). All ionization stages are taken into account, with the exception of the neutral and singly ionized stage of the CNO elements. The occupation numbers are calculated with the assumption of LTE, for a temperature $T=T_{\text {eff }}$ and an electron density $n_{\mathrm{e}}=2.0 \times 10^{14} \mathrm{~cm}^{-3}$, which is typical of regions near the wind base. As the typical electron densities in the outer parts of weak winds are considerably lower and because the assumption of LTE is unreliable in the wind region, an agreement of the derived mass-loss rates with the results of more sophisticated calculations can be expected only if the dependence of the radiative acceleration on the ionization and excitation equilibrium is weak enough.

As in Abbott (1982) the force multiplier can be written as

$M(t)=\sum_{\text {lines }} \frac{F_{v}}{F} \Delta v_{\mathrm{D}} \frac{1-\exp \left(-\eta_{1} t\right)}{t}$,

the sum is over all lines. If $v_{0}$ is the line centre frequency, the Doppler width is defined here according to

$\Delta v_{\mathrm{D}}=\frac{v_{0}}{c} v_{\mathrm{th}}$

Note that $v_{\text {th }}$ is the thermal velocity of hydrogen defined in Eq. (3). With expressions (1) and (22) for $t$ and $\eta_{1}$, it can be seen that in Eq. (19) $v_{\text {th }}$ cancels out.

The ratio $F_{v} / F$ indicates the monochromatic to total flux at the frequency of the line. This flux is calculated from similar models as used in the diffusion calculations e.g. in Unglaub \& Bues (2001). The temperature structure is obtained with the assumption of LTE and with the diffusion approximation for the stellar flux:

$T^{4}=\frac{3}{4} T_{\mathrm{eff}}^{4} \bar{\tau}+T_{0}^{4}$

with $T_{0}^{4}=\frac{1}{2} T_{\text {eff }}^{4}$ at the outer boundary. Here, $\bar{\tau}$ is the Rosseland mean optical depth. The Rosseland mean opacity is calculated 
from the monochromatic continuum opacities for 264 wavelengths in the range $0.1 \AA \leq \lambda \leq 10000 \AA$. Similar to the calculation of the force multipliers, the elements $\mathrm{H}, \mathrm{He}, \mathrm{C}, \mathrm{N}$, and $\mathrm{O}$ are taken into account, while $F_{v}$ is the emergent monocromatic flux of these models at the frequency of the line. It is important to note that only the continuum opacity is taken into account and not the opacity due to lines. Thus it is assumed that each line in the wind "sees" the continuum flux, which is independent of the velocity and thus the Doppler shift. Although this assumption is usual in the CAK theory, it is crucial for the case of thin winds (see Sect. 6.2).

If stimulated emissions are neglected, then $\eta_{1}$ is

$\eta_{\mathrm{l}} \approx \frac{\pi e^{2}}{m_{\mathrm{e}} c} f \frac{n_{i}}{\rho \sigma_{\mathrm{e}} \Delta \nu_{\mathrm{D}}}$

where $e$ is the electron charge, $m_{\mathrm{e}}$ the electron mass, $f$ the oscillator strength of the line, and $n_{i}$ the number density of particles in the lower level. With Eq. (2) for $\sigma_{\mathrm{e}}$, it can be seen that, for fixed degree of ionization and occupation numbers, the quantity $\eta_{1}$ for each line is constant throughout an isothermal wind. As in addition the flux $F_{v}$ is fixed for each line, with these assumptions the force multiplier depends only on $t$.

For $t \rightarrow 0$ the force multiplier converges to its maximum value

$M_{\max }=\sum_{\text {lines }} \frac{F_{v}}{F} \Delta v_{\mathrm{D}} \eta_{1}$.

With expression (22) for $\eta_{1}$ it can be seen that, in this optical thin limit, the strong lines of the most abundant ions preferably contribute to the radiative acceleration. According to Abbott (1982) for small wind optical depth parameters $\log t \lesssim-6$ about $90 \%$ of the total force multiplier originates from only about 100 strong lines. Thus for the calculation of extremely weak winds the small number of lines taken into account in the own force multiplier calculations may be sufficient. For higher wind densities, however, the radiative acceleration and thus the mass-loss rate are underestimated, because in the optically thick limit the contribution of a line is independent of its oscillator strength (e.g. Puls et al. 2000). Then the number of lines taken into account is especially important.

In Fig. 3 for $T_{\text {eff }}=40000 \mathrm{~K}$ and solar metallicity, the force multipliers from own calculations are compared with the ones according to Eq. (25) of Kudritzki (2002) for $n_{\mathrm{e}} / W=10^{14}$ and $10^{10} \mathrm{~cm}^{-3}$, respectively. For the higher values of $t$, the line list and the number of elements taken into account in the own calculations is not sufficient. Thus the corresponding force multipliers are smaller than Kudritzki's ones. For low values of $t$ the results are in good agreement. This shows that the force multipliers obtained with the present assumptions may agree with the results from more sophisticated calculations if the wind optical depth parameter is low enough. However, as the assumption of LTE in the present calculations may be unreliable, this agreement also requires the dependence on the ionization and excitation equilibrium to be small. This is obviously the case in the present example, because the differences between Kudritzki's results for $n_{\mathrm{e}} / W=10^{14}$ and $10^{10} \mathrm{~cm}^{-3}$ decrease to lower values of $t$.

\section{The decoupling of elements}

According to our calculations, the mean radiative acceleration of the metals is by at least two orders of magnitudes higher than of hydrogen and helium. This may justify lumping hydrogen and helium together into one "element" (henceforth element 1) and the metals into "element" 2 , although the velocities of the individual metals are different (Krtička 2006).

A chemically homogeneous, radiatively driven wind may only exist if the coupling due to Coulomb collisions between metals and hydrogen and helium is efficient. If a wind model is calculated from a one-component description it is implicitly assumed that this is the case. Whether this assumption is justified or not may be checked by considering the momentum equations of the individual constituents.

\subsection{Equations for an isothermal three-component model}

For each of the constituents, a momentum equation must be valid:

$\rho_{1} v_{1} \frac{\mathrm{d} v_{1}}{\mathrm{~d} r}+\frac{\mathrm{d} p_{1}}{\mathrm{~d} r}+\rho_{1} \frac{G M_{*}}{r^{2}}-n_{1} Z_{1} e E-\rho_{1} g_{\mathrm{rad}}^{(1)}=\Delta Q_{1}$

$\rho_{2} v_{2} \frac{\mathrm{d} v_{2}}{\mathrm{~d} r}+\frac{\mathrm{d} p_{2}}{\mathrm{~d} r}+\rho_{2} \frac{G M_{*}}{r^{2}}-n_{2} Z_{2} e E-\rho_{2} g_{\mathrm{rad}}^{(2)}=\Delta Q_{2}$.

Here, $\rho_{1}, \rho_{2}, n_{1}, n_{2}$, and $v_{1}, v_{2}$ are the mass densities, particle densities, and mean velocities of "elements" 1 ( $\mathrm{H}$ and $\mathrm{He}$ ) and 2 (metals), respectively, and $g_{\mathrm{rad}}^{(1)}$ and $g_{\mathrm{rad}}^{(2)}$ are the mean radiative accelerations and $Z_{1} e, Z_{2} e$ the mean charges. And $\Delta Q_{1}$ and $\Delta Q_{2}$ are the momentum per unit volume and unit time, which is exchanged between the two constituents via collisions. Because of momentum conservation, it is $\Delta Q_{1}+\Delta Q_{2}=0$. The Coulomb interaction of the elements with the electrons is taken into account via the polarization electric field $E$, which is obtained from the momentum equation for the electrons:

$\rho_{\mathrm{e}} v_{\mathrm{e}} \frac{\mathrm{d} v_{\mathrm{e}}}{\mathrm{d} r}+\frac{\mathrm{d} p_{\mathrm{e}}}{\mathrm{d} r}+\rho_{\mathrm{e}} \frac{G M_{*}}{r^{2}}+n_{\mathrm{e}} e E-\rho_{\mathrm{e}} g_{\mathrm{rad}}^{(\mathrm{e})}=0$

where $g_{\mathrm{rad}}^{(\mathrm{e})}$ is the radiative acceleration on the electrons due to Thomson scattering.

The velocities of the various constituents are related to the centre of mass velocity $v$ according to

$v=\frac{\rho_{1} v_{1}+\rho_{2} v_{2}+\rho_{\mathrm{e}} v_{\mathrm{e}}}{\rho}$.

In addition we demand that there is no net electric current so that the plasma remains neutral on a macroscopic scale:

$Z_{1} e n_{1} v_{1}+Z_{2} e n_{2} v_{2}-e n_{\mathrm{e}} v_{\mathrm{e}}=0$

This system of equations, together with the equations of continuity for each constituent, and the energy equations (if the wind is not assumed to be isothermal) have to be solved to obtain a multicomponent wind model. This has been the approach of Krtička \& Kubát $(2000,2001 a)$. In the present paper for each wind model obtained from the one-component description (from which $\dot{M}$ and $v(r)$ is known), we check whether the momentum transferred from the metals to hydrogen and helium can be sufficient (see Sect. 3.3). Possible heating processes (e.g. frictional heating) are neglected, we assume always $T=T_{\text {eff }}$.

\subsection{The collisional acceleration on $\mathrm{H}$ and $\mathrm{He}$}

A mean collisional acceleration on hydrogen and helium due to collisions with metals may be defined according to

$g_{\mathrm{coll}}^{(1)}=\frac{1}{\rho_{1}} \Delta Q_{1}$. 
From the equations derived by Burgers (1969) it follows that

$g_{\text {coll }}^{(1)}=\rho_{2} k_{12} G(x)$

with

$k_{12}=\frac{1}{m_{1} m_{2}} \frac{4 \pi Z_{1}^{2} Z_{2}^{2} e^{4}}{k_{\mathrm{B}} T} \ln \Lambda$.

With the assumed number ratio $\mathrm{He} / \mathrm{H}=0.1$, the mean mass of $\mathrm{H}$ and $\mathrm{He}$ is $m_{1}=1.27 m_{\mathrm{p}}$. The metals are represented by the CNO elements with abundances proportional to solar ones (the solar abundances are from Grevesse \& Sauval 1998). For the mean mass of the metals, $m_{2}=14.6 m_{\mathrm{p}}$ is assumed. The Coulomb logarithm $\ln \Lambda$ is defined according to Burgers (1969):

$\ln \Lambda=-\frac{1}{2}+\ln \left(\frac{3 k_{\mathrm{B}} T R_{\mathrm{D}}}{Z_{1} Z_{2} e^{2}}\right)$.

Thus, in an isothermal wind with constant ionization, $k_{12}$ is almost constant apart from a weak dependence on density via the Debye radius $R_{\mathrm{D}}$, which appears in the Coulomb logarithm:

$R_{\mathrm{D}}=\left(\frac{k_{\mathrm{B}} T}{4 \pi e^{2}\left(n_{1} Z_{1}^{2}+n_{2} Z_{2}^{2}+n_{\mathrm{e}}\right)}\right)^{0.5}$.

As the particle densities decrease in an outward direction, the Debye radius and thus $\ln \Lambda$ increase. The Chandraskhar function

$G(x)=\frac{1}{2 x^{2}}\left(\operatorname{erf}(x)-x \frac{2}{\sqrt{\pi}} \exp \left(-x^{2}\right)\right)$

depends on the difference in the mean velocities of the constituents. For $v_{2}>v_{1}$ it is

$x=\frac{v_{2}-v_{1}}{\alpha}$

with

$\alpha=\sqrt{\frac{2 k_{\mathrm{B}} T}{m_{12}}}$,

where $\alpha$ has the dimension of a velocity and should not be confused with the force multiplier parameter, and $m_{12}=$ $m_{1} m_{2} /\left(m_{1}+m_{2}\right)$ is the reduced mass. As $m_{12}=1.17 m_{\mathrm{p}}$ in the present case, the quantity $x$ approximately is the velocity difference between both constituents in units of the thermal velocity $v_{\text {th }}$ of hydrogen. The error function $\operatorname{erf}(x)$ has been evaluated with the routines in Press et al. (1992). For $x \ll 1$ the function $G(x)$ increases linearly with $x$. For $x=0.968$, however, it reaches its maximum value

$G_{\max }=0.214$

and decreases to higher values of $x$.

\subsection{Criterion for the existence of a coupled wind}

In a chemically homogeneous wind with a total mass-loss rate $\dot{M}$, the mass-loss rates of the various constituents are by definition (see Sect. 1) $\dot{M}_{1}=\zeta_{1} \dot{M}$ for hydrogen and helium and $\dot{M}_{2}=\zeta_{2} \dot{M}$ for the metals. Then the equations of continuity for both constituents can be written as

$\zeta_{1} \dot{M}=4 \pi r^{2} \rho_{1} v_{1}$

$\zeta_{2} \dot{M}=4 \pi r^{2} \rho_{2} v_{2}$
The mass fractions $\zeta_{1}$ and $\zeta_{2}$ of hydrogen and helium and metals specify the metallicity of the star. For solar composition we use $\zeta_{2}=0.0133$. As $\dot{M}$ is the total mass-loss rate, these continuity equations state that the relative mass flows of the constituents correspond to their relative mass fractions in the stellar atmosphere. Only if this is true can the surface composition of the star be expected to remain unchanged.

In the present paper, metals are considered as trace elements so that $\rho_{2} \ll \rho_{1}$. As it is $\rho_{\mathrm{e}} \ll \rho_{1}$, it follows that $v_{1} \approx v$ from Eq. (27). The velocity $v_{2}$ of the mean metal must be higher than $v_{1}$ for that momentum is given from the metals to hydrogen and helium. Thus, with $v_{2}=v$, Eq. (39) yields an upper limit for the mass density $\rho_{2}$. With this value and with $G(x)=G_{\max }$, from Eq. (30) an upper limit for the mean collisional acceleration on hydrogen and helium can be derived:

$g_{\mathrm{coll}}^{(\max )}=\frac{\zeta_{2} \dot{M}}{4 \pi r^{2} v} k_{12} G_{\max }$.

For each wind model obtained from a one-component description and with $v_{1} \approx v$ and $\rho_{1} \approx \rho$ the lefthand side of the momentum equation for hydrogen and helium (Eq. (24)) is known at each grid point. Then the constituents may be coupled only if

$g_{\text {coll }}^{(\max )} \geq v \frac{\mathrm{d} v}{\mathrm{~d} r}+\frac{1}{\rho} \frac{\mathrm{d} p_{1}}{\mathrm{~d} r}+\frac{G M_{*}}{r^{2}}-\frac{1}{m_{1}} Z_{1} e E-g_{\mathrm{rad}}^{(1)}$.

If this condition is not fulfiled eyerywhere in the wind, then the wind solution is incompatible with the assumption that the wind is chemically homogeneous. In this case clearly a multicomponent description would be required. The mean radiative acceleration $g_{\text {rad }}^{(1)}$ on hydrogen and helium is obtained from own calculations with assumptions as described in Sect. 2.4.2. The results have shown that its effect is negligibly small. In Sects. 4 and 5, mass-loss rates are predicted as a function of surface gravity. If $g_{\text {rad }}^{(1)}$ were neglected in criterion (41), the maximal surface gravity up to which coupled winds can exist would be lower by about 0.05 dex only in $\log g$.

\subsection{Comparison with criteria from other authors}

In condition (41) the contribution of the gas pressure to the momentum equation is taken into account. In the supersonic region, where this contribution is small, criterion (41) reduces to the one derived e.g. by Owocki \& Puls (2002). If in Eq. (26) all terms proportional to $\rho_{\mathrm{e}}$ are neglected, it follows that

$E \approx-\frac{1}{e n_{\mathrm{e}}} \frac{\mathrm{d} p_{\mathrm{e}}}{\mathrm{d} r}$

With this approximation the radiative acceleration $g_{\text {rad }}^{(\mathrm{e})}$ due to the light scattering on free electrons has been neglected. This is justified in stars with $\Gamma_{\mathrm{e}} \ll 1$ (see Sect. 2). If the metals are trace elements, it is $n_{\mathrm{e}} \approx Z_{1} n_{1}$. With $v_{1} \approx v$, from Eq. (28), it follows that $v_{\mathrm{e}} \approx v$. Thus hydrogen and helium, as well as the free electrons, move approximately with the centre of mass velocity $v$. If approximation (42) is inserted into condition (41), then with $p \approx p_{1}+p_{\mathrm{e}}$ the latter can be written as

$g_{\mathrm{coll}}^{(\max )} \geq v \frac{\mathrm{d} v}{\mathrm{~d} r}+\frac{G M_{*}}{r^{2}}+\frac{1}{\rho} \frac{\mathrm{d} p}{\mathrm{~d} r}-g_{\mathrm{rad}}^{(1)}$.

If the contributions of the gas pressure and of the radiative force on hydrogen and helium are neglected, this criterion is equivalent to the one in Owocki \& Puls (2002). This is a good approximation in the supersonic region, whereas the criterion used in the 
present paper may also be applied in the subsonic region, where the contribution of the gas pressure is essential. If the acceleration term $v \mathrm{~d} v / \mathrm{d} r$ is also neglected, it follows that

$g_{\text {coll }}^{(\max )} \geq \frac{G M_{*}}{r^{2}}$

In the supersonic region, this is a necessary but not sufficient condition for the existence of a coupled wind. It only requires that hydrogen and helium do not decelerate. In wind models obtained from the improved version of the CAK theory, the acceleration term should be more important than the gravitational one (Gayley 2000). In the present wind models for high gravity stars obtained from the original version of the CAK theory, both terms have a similar order of magnitude in the supersonic region.

For a given mass-loss rate and metallicity, with Eq. (40) for $g_{\text {coll }}^{(\max )}$, a maximum velocity can be obtained up to which criterion (44) may be fulfiled:

$v_{\max }=\frac{\zeta_{2} \dot{M}}{4 \pi G M_{*}} k_{12} G_{\max }$.

This $v_{\max }$ is an upper limit of the velocity, up to which the constituents may be coupled. In accelerating winds decoupling should in fact occur at lower velocities. Equation (45) corresponds to Eq. (22) of Owocki \& Puls (2002) for the case $w=0$ in their notation.

This maximum velocity may be compared with the surface escape velocity:

$v_{\mathrm{esc}}=\sqrt{\frac{2 G M_{*}\left(1-\Gamma_{\mathrm{e}}\right)}{R_{*}}}$.

For $\Gamma_{\mathrm{e}} \ll 1$, with $R_{*}=\sqrt{G M_{*} g^{-1}}$, it follows that

$v_{\mathrm{esc}}=\sqrt{2}\left(G M_{*} g\right)^{\frac{1}{4}}$.

With the definition

$y=\frac{n_{2}}{n_{1}}$

and $\zeta_{2} \approx m_{2} m_{1}^{-1} y$, with Eq. (31) for $k_{12}$, the ratio $v_{\max } / v_{\text {esc }}$ can be written as

$\frac{v_{\max }}{v_{\mathrm{esc}}}=\dot{M} y \frac{Z_{1}^{2} Z_{2}^{2} e^{4} \ln \Lambda G_{\max }}{m_{1}^{2} k_{\mathrm{B}} T} \frac{1}{\sqrt{2}}\left(G M_{*}\right)^{-\frac{5}{4}} g^{-\frac{1}{4}}$.

For the wind models considered here, the Coulomb logarithm $\ln \Lambda$ varies between about 6.0 at the wind base and 18.0 in the outer regions of the thinnest winds. With the values assumed by Owocki \& Puls (2002), $\ln \Lambda=20, Z_{1}=1, m_{1}=m_{\mathrm{p}}$, for a stellar mass $M_{*}=0.5 M_{\odot}$ as considered here, it is

$\frac{v_{\max }}{v_{\text {esc }}}=1.4 \times 10^{20} \dot{M} y Z_{2}^{2} T^{-1} g^{-\frac{1}{4}}$

where the mass-loss rate $\dot{M}$ is in $M_{\odot} / \mathrm{yr}$, and the surface gravity $g$ in $\mathrm{cm} \mathrm{s}^{-2}$. The temperature $T$ (in $\mathrm{K}$ ) in the present paper is assumed to be equal to the effective temperature of the star. Frictional heating could reduce $v_{\max }$ and thus favour decoupling, if the effect of a higher temperature is not compensated by a higher mean charge of the metals.

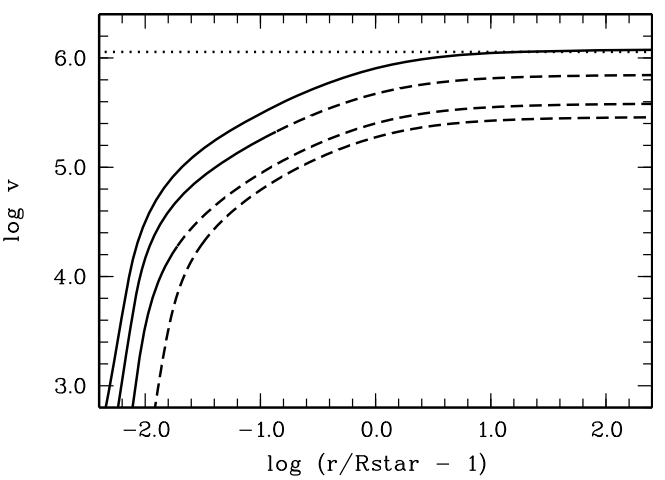

Fig. 4. Solutions $v(r)$ (in $\mathrm{m} \mathrm{s}^{-1}$ ) for $T_{\text {eff }}=40000 \mathrm{~K}, \log g=5.8$, $M_{*}=0.5 M_{\odot}$, and (from top to the bottom) $Z / Z_{\odot}=1,1 / 3,0.2$, and 0.1 . Dashed lines indicate decoupling of metals, the dotted line represents the surface escape velocity.

Table 1. Predicted mass-loss rates (in $M_{\odot} / \mathrm{yr}$ ), terminal velocities, and velocities at which decoupling is expected according to criteria (41) and (50), respectively, for $T_{\text {eff }}=40000 \mathrm{~K}, \log g=5.8$, and various metal abundances. The surface escape velocity is $v_{\mathrm{esc}}=1136 \mathrm{~km} \mathrm{~s}^{-1}$.

\begin{tabular}{lllll}
\hline \hline$Z / Z_{\odot}$ & $\log \dot{M}$ & $v_{\infty} / v_{\mathrm{esc}}$ & $v_{\mathrm{d}} / v_{\mathrm{esc}}(41)$ & $v_{\max } / v_{\mathrm{esc}}(50)$ \\
\hline 1.0 & -11.3 & 1.10 & - & 5.64 \\
$1 / 3$ & -11.9 & 0.63 & 0.186 & 0.47 \\
$1 / 5$ & -12.9 & 0.35 & 0.017 & 0.028 \\
$1 / 10$ & -14.7 & 0.26 & 0.0004 & 0.0002 \\
\hline
\end{tabular}

\subsection{Example: $T_{\text {eff }}=40000 \mathrm{~K}, \log g=5.8$}

For $T_{\text {eff }}=40000 \mathrm{~K}, \log g=5.8, M_{*}=0.5 M_{\odot}$, and several metal abundances, the solutions $v(r)$ are shown in Fig. 4. It is indicated in which parts of the solution condition (41) is fulfiled, and the maximum velocities $v_{\mathrm{d}}$ up to which this is the case are given in Table 1. In these calculations the metals are represented by the $\mathrm{CNO}$ elements with force multipliers from own calculations as described in Sect. 2.4.2.

The only case where the constituents may be coupled throughout the wind is for $Z / Z_{\odot}=1$, for which a mass-loss rate $\log \dot{M}=-11.3$ is predicted. (This value agrees with the one obtained in Sect. 4 with the force multipliers from Kudritzki 2002) This mass-loss rate is lower by a factor of $\sim 1000$ than in the main sequence star $\tau$ Sco, for which Springmann \& Pauldrach (1992) used the values $\dot{M}=5 \times 10^{-9} M_{\odot} / \mathrm{yr}, M_{*}=19.6 M_{\odot}$, $R_{*}=5.5 R_{\odot}$ and $T_{\text {eff }}=33000 \mathrm{~K}$ in their analysis of multicomponent effects, which corresponds to $\log g=4.3$. As in the present example the stellar radius is only $R_{*}=0.15 R_{\odot}$, the mass flux $\dot{M} /\left(4 \pi R_{*}^{2}\right)$ near the stellar surface has a similar order of magnitude to the one in $\tau$ Sco.

Thus the wind densities are similar. Springmann \& Pauldrach (1992) and Krtička \& Kubát (2001b) find that in $\tau$ Sco the multicomponent effects are important due to their contribution to the energy equation. In the present example, the relative velocities between metals and the passive plasma are up to $7 \mathrm{~km} \mathrm{~s}^{-1}$. This may lead to frictional heating. Thus, as in $\tau$ Sco, the present example is near the border of the runaway condition. Multicomponent effects may have some importance; however, the coupling should still be effective enough the passive plasma to be expelled from the star.

If the metal abundance is reduced by a factor of three, $\log \dot{M}=-11.9$ is predicted by the one-component wind model, which is lower by a factor of four as in the case with $Z / Z_{\odot}=$ 1. Because of the reduced mass-loss rate and the additional 

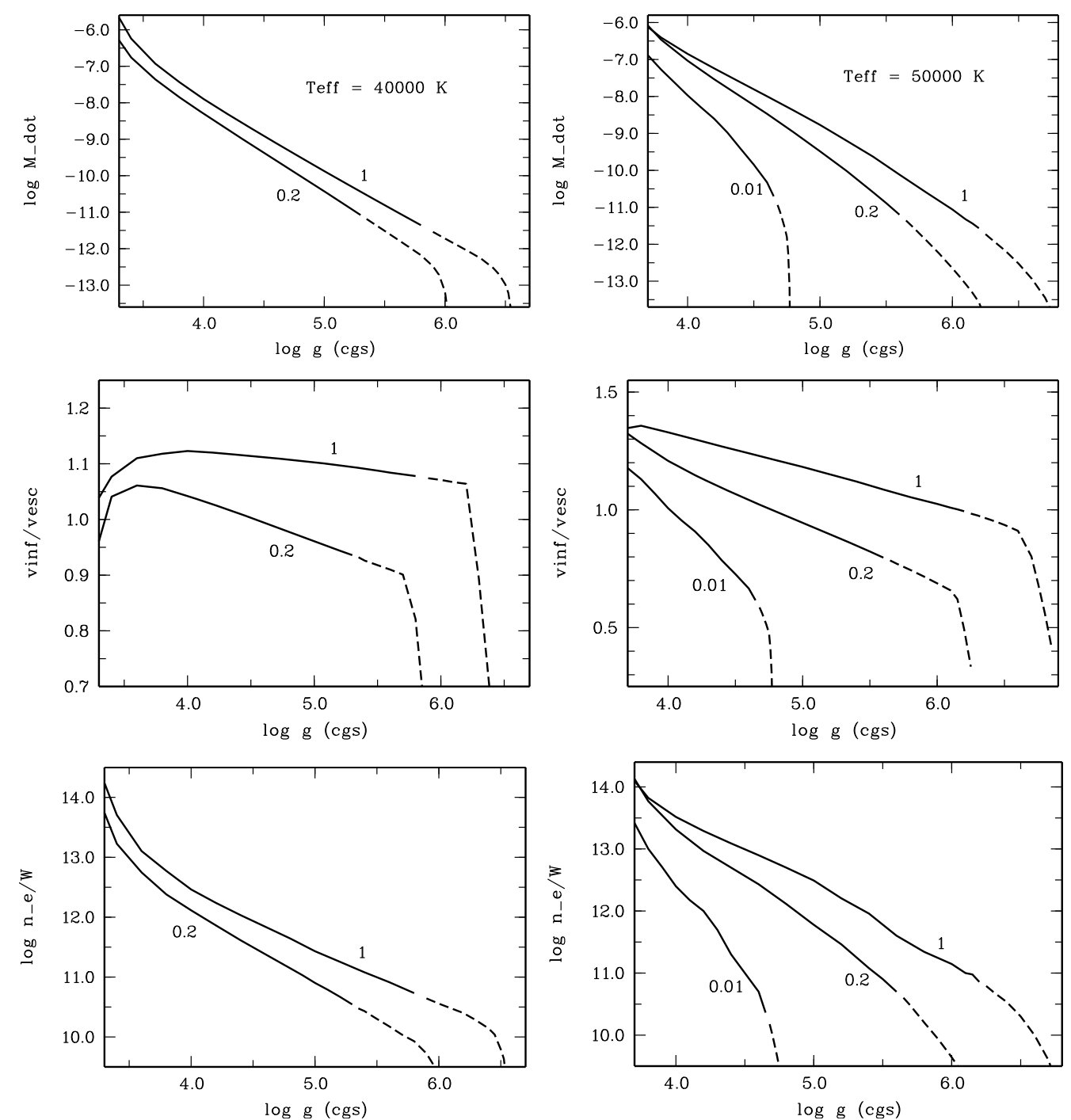

Fig. 5. Left: predicted mass-loss rates (in $M_{\odot} / \mathrm{yr}$ ), terminal velocities, and the adopted values of $n_{\mathrm{e}} / W\left(\right.$ in $\mathrm{cm}^{-3}$ ) as a function of the surface gravity for $T_{\text {eff }}=40000 \mathrm{~K}, M_{*}=0.5 M_{\odot}$, and $Z / Z_{\odot}=1$ and 0.2 , respectively. Right: the same for $T_{\text {eff }}=50000 \mathrm{~K}$ and $Z / Z_{\odot}=1,0.2$, and 0.01 . The results for which decoupling of the metals is expected are represented by dashed lines.

reduction of the metal abundance, the density of metals in the wind effectively is lower by a factor of 12 . Therefore in this case decoupling is expected at a velocity $v_{\mathrm{d}}=210 \mathrm{~km} \mathrm{~s}^{-1}$ and at a radius $r=1.13 R_{*}$. As the decoupling occurs near the stellar surface at a velocity that is lower by about a factor of five than the surface escape velocity $v_{\text {esc }}=1136 \mathrm{~km} \mathrm{~s}^{-1}$, the passive plasma cannot be expelled from the star. For metal abundances reduced by more than a factor of five with predicted mass-loss rates $\dot{M} \lesssim 10^{-13} M_{\odot} / \mathrm{yr}$, the decoupling is even expected in the subsonic region.

If the stellar parameters and the calcuated mass-loss rates are inserted into Eq. (50), with a solar number ratio of metals to hydrogen and helium $y_{\odot} \approx 10^{-3}$, it can be seen that this criterion for ion decoupling leads to similar conclusions. The values of $v_{\max } / v_{\text {esc }}$ are given in the last column of Table 1 . It is $v_{\max }>v_{\infty}$ only for solar metallicity. For the cases with reduced metallicity, it is $v_{\max }<v_{\infty}$, although criterion (50) overestimates $v_{\max }$. Thus it follows that, at least for the cases with reduced metallicity, no coupled wind can exist. This could only be possible, if the terminal velocities were significantly lower than predicted from the present calculations.
Krtička \& Kubát (2000) suggest that in some cases decoupling could be avoided if the wind switches to a solution with an abrupt lowering of the velocity gradient. However, the physical relevance of such solutions has been questioned by Owocki \& Puls (2002) and Krtička \& Kubát (2002), who argue that they are unstable. The time-dependent hydrodynamical calculations of Votruba et al. (2007) show that the ions decouple and are accelerated to high velocities, whereas the passive plasma decelerates.

Feldmeier \& Nikutta (2006) investigated the effect of radiative coupling between distant locations in winds with nonmonotonic velocity laws and allowed for wind deceleration. They find that this could lead to a lowering of the terminal velocity, which may be below the surface escape velocity by a factor of the order two to three. However, the existence of chemically homogeneous winds with $\dot{M} \approx 10^{-13} M_{\odot} / \mathrm{yr}$, which have been assumed e.g. by Unglaub \& Bues (2001) to explain the helium abundances in sdB stars, would require the existence of winds with $v_{\infty} \approx 0.1 v_{\text {esc }}$. Such a strong lowering of the terminal velocity seems to be unlikely. As discussed in Sect. 6 from the various simplifications used in the present calculations, it should instead 
Table 2. Adopted electron scattering opacities and mean charges $Z_{1}$ (hydrogen and helium) and $Z_{2}$ (metals).

\begin{tabular}{cccc}
\hline \hline$T_{\text {eff }}[K]$ & $\sigma_{\mathrm{e}}\left[\mathrm{cm}^{2} \mathrm{~g}^{-1}\right]$ & $Z_{1}$ & $Z_{2}$ \\
\hline 40000 & 0.33 & 1.08 & 2.95 \\
50000 & 0.34 & 1.09 & 3.51 \\
\hline
\end{tabular}

be expected that the terminal velocities are underestimated. Then the mass-loss rates required for the existence of a coupled wind would be higher.

\section{Results for $\mathrm{T}_{\text {eff }}=\mathbf{4 0 0 0 0}$ and $50000 \mathrm{~K}$}

This section presents the mass-loss predictions as a function of the surface gravity for $T_{\mathrm{eff}}=40000 \mathrm{~K}$ and $50000 \mathrm{~K}$ and for $M_{*}=0.5 M_{\odot}$ (see Fig. 5). The radiative acceleration is obtained with the force multipliers from Kudritzki (2002) for several metallicities. The adopted values of $n_{\mathrm{e}} / W$ are shown in the lower panels, which were obtained according to the iteration procedure described in Sect. 2.4.1. For each wind model, it was checked whether decoupling of metals from hydrogen and helium is expected according to criterion (41); the adopted mean charges of these constituents are given in Table 2 . The results of the calculations for which the actual one component description of the wind may be sufficient, are represented by solid lines.

The surface gravities for which wind models were calculated range from values where the star would be near the Eddington limit $\left(\Gamma_{\mathrm{e}} \approx 0.8\right)$ to values for which no wind solution exists.

For $T_{\text {eff }}=40000 \mathrm{~K}, Z / Z_{\odot}=1$, coupled winds can exist up to $\log g=5.8$ with a mass-loss rate of $\dot{M}=4.4 \times 10^{-12} M_{\odot} / \mathrm{yr}$. For $Z / Z_{\odot}=0.2$, this is only the case for $\log g \leq 5.3$. Due to the higher luminosities for $T_{\text {eff }}=50000 \mathrm{~K}$, the mass-loss rates for given $\log g$ and $Z$ are higher than for $T_{\text {eff }}=40000 \mathrm{~K}$. However, even for solar metallicity, chemically homogeneous winds can exist for $\log g \leq 6.2$ alone. Thus for DA white dwarfs in the considered range of effective temperatures, their existence can be excluded. For $\log g>7.0$ no wind solution exists at all, because the radiative acceleration is too low even for wind optical depth parameters $t \rightarrow 0$. For $T_{\text {eff }}=50000 \mathrm{~K}, Z / Z_{\odot}=0.2$, and $Z / Z_{\odot}=0.01$, we expect the existence of coupled winds for $\log g \leq 5.5$ and for $\log g \leq 4.6$, respectively.

The results show an increasing dependence of the mass-loss rates on metallicity to higher gravities. For $T_{\text {eff }}=40000 \mathrm{~K}$, $\log g=4.0$, and $Z / Z_{\odot}=1$, a mass-loss rate of $\dot{M}=1.3 \times$ $10^{-8} M_{\odot} / \mathrm{yr}$ is predicted. For $Z / Z_{\odot}=0.2$ and the same stellar parameters, it is $\dot{M}=5.0 \times 10^{-9} M_{\odot} /$ yr. From a comparison of these two values, a dependence of the mass-loss rate on the metallicity according to $\dot{M} \sim Z^{0.58}$ may be suggested. For $\log g=5.0$ and the same effective temperature the mass-loss rates for the two metallicities are $1.3 \times 10^{-10}$ and $3.7 \times 10^{-11} M_{\odot} / \mathrm{yr}$, respectively. From these values it would follow that $\dot{M} \sim Z^{0.80}$. This shows that, for weak winds, the $Z$-dependence cannot be represented by a power law of the form $\dot{M} \sim Z^{\epsilon}$ with constant exponent $\epsilon$. For the present example, $\epsilon$ would increase from $\epsilon \approx 0.6$ for the lowest gravities up to values $\epsilon>1$ for $\log g \gtrsim 5.5$. This effect can still be seen more clearly for the case $T_{\text {eff }}=50000 \mathrm{~K}$ in Fig. 5 , for which results for $Z / Z_{\odot}=0.01$ are also shown. This breakdown of the usual scaling relations for sufficiently weak winds has already been found by Kudritzki (2002) in his calculations for stars with extremely low metallicities.

From the middle panels of Fig. 5 it can be seen that the terminal velocities of the present wind models have a similar order of magnitude to the surface escape velocities. This is expected
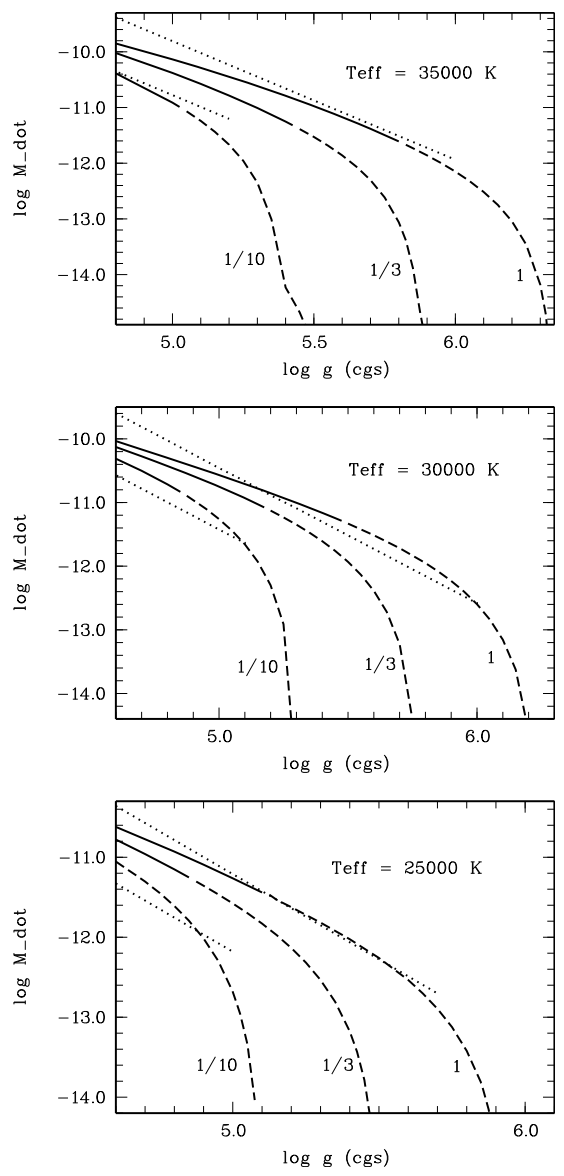

Fig. 6. Predicted mass-loss rates (in $M_{\odot} / \mathrm{yr}$ ) as a function of surface gravity for $T_{\text {eff }}=35000,30000,25000 \mathrm{~K}$ and $Z / Z_{\odot}=1,1 / 3,0.1$ with $M_{*}=0.5 M_{\odot}$. Dashed lines indicate decoupling of metals in the corresponding wind model. The upper and lower dotted lines in each figure represent the results of Vink \& Cassisi (2002) for $Z / Z_{\odot}=1$ and 0.1 , respectively.

from the original version of the CAK theory, as used in the present paper. According to the improved version of the theory they should be larger (see Sect. 6.1). With $M(t)=k t^{-\alpha}$, it follows from the original theory that $v_{\infty}=\sqrt{\frac{\alpha}{1-\alpha}}$ (see e.g. Lamers $\&$ Cassinelli 1999). For $\alpha$ between about 0.5 and 0.7 , ratios of $1.0 \lesssim v_{\infty} / v_{\text {esc }} \lesssim 1.5$ should be expected. As for small wind optical depth parameters the $\log M(t)-\log t$ relation flattens and its slope $-\alpha$ finally approaches zero, the predicted ratio $v_{\infty} / v_{\text {esc }}$ may be lower for weak winds, however. The sudden decrease of $v_{\infty} / v_{\text {esc }}$ and in the mass-loss rates, which occur in some cases near the highest gravities for which a wind solution still exists (e.g. for $T_{\text {eff }}=40000 \mathrm{~K}, Z / Z_{\odot}=1$ near $\log g=6.3$ ), are a consequence of the restriction of Kudritzki's force multipliers to a value $M_{\max }$ as described in Sect. 2.4.1. The results for lower gravities, however, are not affected by this restriction.

The results of this section show that the existence of coupled winds requires mass-loss rates at least of the order $10^{-11} M_{\odot} / y r$. For stars with $M_{*}=0.5 M_{\odot}$, effective temperatures in the range $40000 \mathrm{~K} \leq T_{\text {eff }} \leq 50000 \mathrm{~K}$, and solar metallicity, this is possible only for surface gravities $\log g \lesssim 6.0$. Stars with stellar parameters within these ranges may be pre-white dwarfs, which are in an evolutionary stage between the asymptotic giant branch or the EHB and the white dwarf cooling sequence. Thus, if the metallicity is not too far below the solar value, the mass-loss rates should be high enough to prevent the effect of diffusion. 


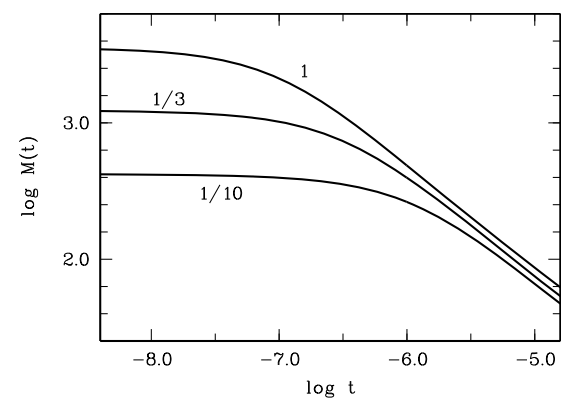

Fig. 7. Force multipliers according to our calculations as a function of the wind optical depth parameter for $T_{\text {eff }}=30000 \mathrm{~K}$ and $Z / Z_{\odot}=1,1 / 3$, and 0.1 .

Table 3. Adopted electron scattering opacities $\sigma_{\mathrm{e}}$, and mean charges $Z_{1}$ of hydrogen and helium and $Z_{2}$ of the metals, respectively.

\begin{tabular}{ccccc}
\hline \hline$T_{\text {eff }}[K]$ & $Z / Z_{\odot}$ & $\sigma_{\mathrm{e}}\left[\mathrm{cm}^{2} \mathrm{~g}^{-1}\right]$ & $Z_{1}$ & $Z_{2}$ \\
\hline 35000 & 1 & 0.32 & 1.03 & 2.60 \\
35000 & $1 / 3$ & 0.32 & 1.03 & 2.58 \\
35000 & $1 / 10$ & 0.32 & 1.03 & 2.58 \\
30000 & 1 & 0.31 & 1.00 & 2.15 \\
30000 & $1 / 3$ & 0.31 & 1.00 & 2.15 \\
30000 & $1 / 10$ & 0.31 & 1.00 & 2.15 \\
25000 & 1 & 0.31 & 1.00 & 1.98 \\
25000 & $1 / 3$ & 0.31 & 1.00 & 1.98 \\
25000 & $1 / 10$ & 0.31 & 1.00 & 1.98 \\
\hline
\end{tabular}

In no case can chemically homogeneous winds with $\dot{M} \lesssim$ $10^{-12} M_{\odot} / \mathrm{yr}$ exist, for which the effect of diffusion would become effective according to e.g. Unglaub \& Bues (2001). That this is hardly possible can already be seen from Eq. (50). If e.g. for $T_{\text {eff }}=40000 \mathrm{~K}, \log g=6.0$ and $y=10^{-3}$ (which approximately corresponds to solar metallicty) a mass-loss rate $\dot{M}=10^{-12} M_{\odot} / \mathrm{yr}$ is inserted; then with $Z_{2} \approx 3.0$, it follows that $v_{\mathrm{max}} / v_{\mathrm{esc}}=1$. Thus in winds with terminal velocities $v_{\infty} \gtrsim v_{\mathrm{esc}}$, which should be expected at least according to the improved version of the CAK theory (see Sect. 6), it is $v_{\max }<v_{\infty}$, so the constituents cannot be coupled throughout the wind.

\section{Results for sdB stars}

In Fig. 6 for $T_{\text {eff }}=35000 \mathrm{~K}, 30000 \mathrm{~K}, 25000 \mathrm{~K}, Z / Z_{\odot}=1$, $1 / 3,0.1$, and for $M_{*}=0.5 M_{\odot}$, the predicted mass-loss rates are shown as a function of the surface gravity. These results have been obtained with force multipliers from our own calculations (see Sect. 2.4.2). The adopted values for the electron scattering opacity and for the mean charges of hydrogen and helium and for the metals are given in Table 3.

For the typical range of surface gravities of sdB stars $(5.0 \lesssim$ $\log g \lesssim 6.0$ ), the predicted mass loss rates are between about $10^{-10} M_{\odot} / \mathrm{yr}$ and zero. The results strongly depend on surface gravity and metallicity. At least for such cases with $\dot{M} \lesssim 3 \times$ $10^{-12} M_{\odot} / \mathrm{yr}$, decoupling of the metals from the passive plasma is expected. Thus the mass-loss rates may be high enough to prevent decoupling, but only in the most luminous sdB stars. For the more compact sdB stars from the one-component description of the wind mass-loss rates between about $10^{-12} M_{\odot} / \mathrm{yr}$ and zero are predicted. However, the constituents cannot be coupled in such weak winds.

Vink \& Cassisi (2002) derived mass-loss rates from the requirement of a global momentum balance with the assumption of a $\beta$-type velocity law with $v_{\infty}=v_{\text {esc }}$. They calculated the

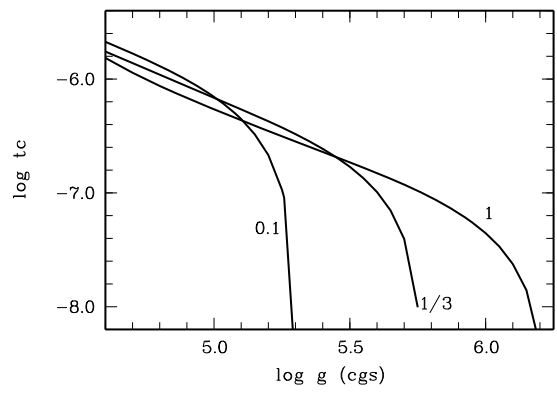

Fig. 8. Critical wind optical depth parameters as a function of the surface gravity for $T_{\text {eff }}=30000 \mathrm{~K}$ and $Z / Z_{\odot}=1,1 / 3$ and 0.1 .

radiative acceleration by means of a Monte Carlo simulation including about $10^{5}$ of the strongest lines of the elements $\mathrm{H}-\mathrm{Zn}$ with NLTE occupation numbers for the most important elements. As can be seen from Fig. 6, especially for $Z / Z_{\odot}=1$ and for $5.0 \lesssim \log g \lesssim 6.0$, their results are in good agreement with the present ones. This may appear surprising, because Vink \& Cassisis's calculations of the radiative force are clearly more sophisticated. This point will be discussed in more detail in Sect. 5.1 for the case $T_{\text {eff }}=30000 \mathrm{~K}$.

The steep decrease in the mass-loss rates for $Z / Z_{\odot}=0.1$ and $\log g \gtrsim 5.0$ does not contradict Vink \& Cassisi's predictions, as it may seem if their results for more luminous stars were extrapolated to higher gravities. Calculations of Vink (priv. comm.) for cases with $\log g=5.5$ and $Z / Z_{\odot}=0.1$ have shown that no mass loss rate exists for which a global momentum balance can be fulfiled. Thus, if multicomponent effects are neglected, the mass-loss rate must approach zero for $\log g<5.5$.

\subsection{Detailed discussion for $T_{\text {eff }}=30000 \mathrm{~K}$}

In Fig. 7 for $T_{\text {eff }}=30000 \mathrm{~K}$ and $Z / Z_{\odot}=1,1 / 3$, and 0.1 , the force multipliers according to own calculations are plotted as a function of the wind optical depth parameter. The mass-loss rates shown in the middle panel of Fig. 6 were calculated with these force multipliers. The corresponding critical wind optical depth parameters (see Sect. 2.1) are shown in Fig. 8. It can be seen that, for the considered surface gravities $4.6 \leq \log g \leq$ 6.2 , they are in the range $-5.5>\log t_{\mathrm{c}} \gtrsim-8.0$. As can be seen from Fig. 7, the slope $-\alpha$ of the $\log M(t)-\log t$ relation is not constant in this range of wind optical depth parameters and $\alpha$ decreases to lower values of $t$. Thus, in a scaling law according to $\dot{M} \sim L^{\frac{1}{\alpha}}$ (Puls et al. 2000), the exponent varies. An increasing dependence of the mass-loss rate on the luminosity to thinner winds is expected. In the present calculations for fixed $T_{\text {eff }}$ and $M_{*}$, this explains why the dependence of the mass-loss rate on the surface gravity increases to higher values of $\log g$, as can be seen from the results in Fig. 6.

In Fig. 9 for $T_{\text {eff }}=30000 \mathrm{~K}$ and $Z / Z_{\odot}=1$ and 0.1 , the contributions of the various elements taken into account in the calculations presented in this section to the force multiplier are plotted as a function of the wind optical depth parameter. It can be seen that in all cases the major contribution is due to carbon for low values of $t$, which are relevant in the present calculations. From the present LTE assumption, it follows that $58 \%$ of all carbon is C III and $42 \%$ is C IV. The contribution of other ionization states of carbon is negligible.

In Fig. 10 for $Z / Z_{\odot}=1$, the contributions of the lines $\mathrm{C}$ III $\lambda 977.0 \AA, \lambda 1175.7 \AA$ and of C IV $\lambda 1549.1 \AA$ to the force multiplier are shown as a function of $t$. At $\log t=-6.0$, about $50 \%$ 

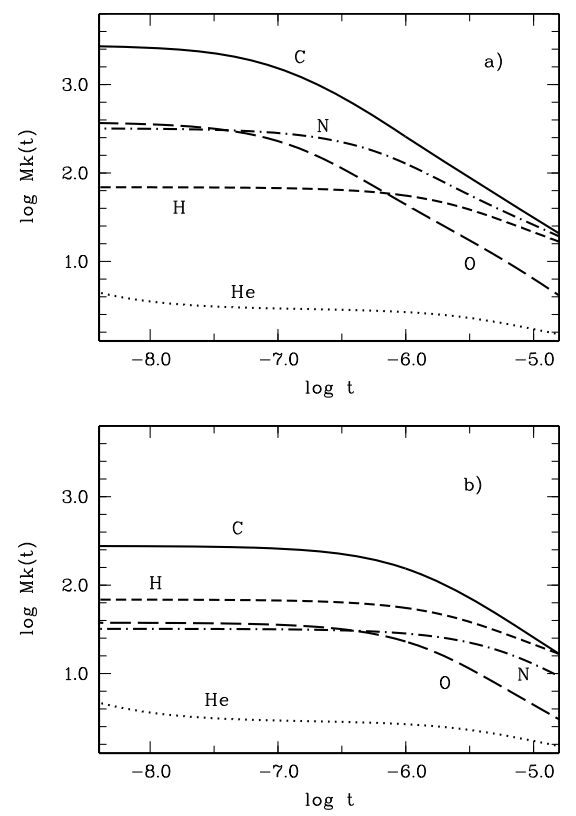

Fig. 9. Force multipliers due to the elements $\mathrm{H}, \mathrm{He}, \mathrm{C}, \mathrm{N}$, and $\mathrm{O}$ for $T_{\text {eff }}=30000 \mathrm{~K}$ and for a) solar abundances, b) abundances of C, N, and $\mathrm{O}$ reduced by a factor of ten.

of the total force multiplier is due to these three lines alone. For $t \rightarrow 0$ this contribution increases to $77 \%$. The most important one of these three lines, especially for $t \rightarrow 0$, is CIII $\lambda 977 \AA$.

Now let us discuss the special example $T_{\text {eff }}=30000 \mathrm{~K}$, $\log g=5.5$. A coupled wind with a mass-loss rate $\dot{M}=4.7 \times$ $10^{-12} M_{\odot} / y r$ is predicted. The critical wind optical depth parameter is $\log t_{\mathrm{c}}=-6.7$. Here the contribution of these three carbon lines to the total force multiplier is $67 \%$. If all elements other than carbon were neglected, the derived mass-loss rate would be lower only by a factor of two $\left(\dot{M}=2.5 \times 10^{-12} M_{\odot} / \mathrm{yr}\right)$. The effect of uncertainties in the CIII / CIV ionization equilibrium have a similar order of magnitude. If it were assumed that all carbon is in the ground state of C III, so that $\lambda 977 \AA$ is the only line to significantly contribute to the radiative force, this again would lead to $\dot{M}=2.5 \times 10^{-12} M_{\odot} / y r$. If on the other hand it is assumed that all carbon is in the ground state of $\mathrm{C} \mathrm{IV}$, this would reduce the mass loss rate by a factor of 3.6 to $\dot{M}=1.3 \times 10^{-12} M_{\odot} / \mathrm{yr}$. These results may explain the agreement between the present predictions and Vink \& Cassisi's within a factor of about two. As the major contribution to the radiative force is due to a few lines of carbon, the number of other lines and elements taken into account is not essential. Secondly, the predicted mass-loss rates on the other hand always have the same order of magnitude, independent of the C III / CIV ionization equilibrium. If the ionization equilibrium changes, then the next ionization state takes over the contribution to the radiative force.

If the metal abundances are reduced by a factor of ten, then the major contribution to the force multiplier is still due to carbon (Fig. 9b). According to the present LTE calculations, the second largest contribution is due to hydrogen. For a wind optical depth parameter $\log t=-6.0$, about $71 \%$ of this contribution is due to the line $\mathrm{L} \alpha$. Thus it essentially depends on the number density of the particles that are in the ground state of HI. The results shown in Fig. 9 were obtained with a number ratio of these particles to all hydrogen particles $\mathrm{HI}(n=$ $1) /(\mathrm{HI}+\mathrm{HII})=7.5 \times 10^{-6}$. If the HI/HII ionization equilibrium is shifted to HII, then, in contrast to the CIII/CIV ionization equilibrium, the next ionization state cannot take over. Thus a strong

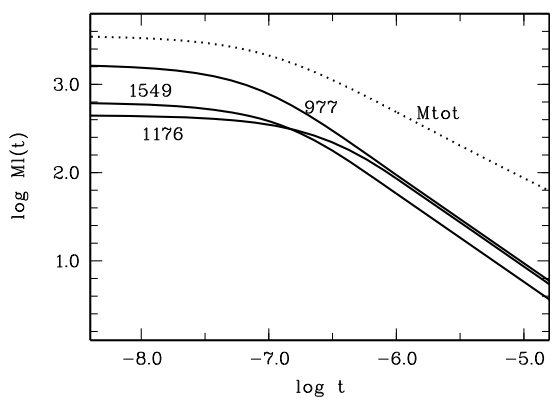

Fig. 10. Force multipliers due to the lines CIII $\lambda 977.0$ and $\lambda 1175.7 \AA$ and CIV $\lambda 1549.1 \AA$ for $T_{\text {eff }}=30000 \mathrm{~K}$ and solar abundances. The dotted line represents the total force multiplier.

dependence on the ionization equilibrium is expected and the present results for hydrogen are questionable, because NLTE effects have not been taken into account. As the major contribution to the force multiplier for $Z / Z_{\odot}=0.1$ is still due to carbon, however, the predicted mass-loss rates still agree approximately with the ones of Vink \& Cassisi (2002). A possible overestimate of the relative contribution of hydrogen to the force multiplier may be the reason that, for the cases with $\log g \lesssim 5.0$ shown in Fig. 6, the dependence of the mass-loss rates on the metallicity is somewhat less than in Vink \& Cassisi's results.

For $T_{\text {eff }}=25000 \mathrm{~K}$ the situation is similar to $T_{\text {eff }}=$ $30000 \mathrm{~K}$. The major contribution to the radiative force is due to carbon and the line C III $\lambda 977 \AA$. For $T_{\text {eff }}=35000 \mathrm{~K}$ and wind optical depth parameters $\log t \lesssim-6.0$ according to the present results, carbon is still the most important element. However, the contributions of $\mathrm{N}$ and $\mathrm{O}$ to the force multiplier are only slightly lower, by less than a factor of two.

\section{Discussion}

In Sects. 6.1 and 6.2 we discuss the effects of the omission of the finite disk correction factor, of neglecting changes in ionization in the wind, and of the shadowing of the flux by the photospheric lines. Possible consequences of the results for the surface composition of the various types of chemically peculiar stars are then discussed in Sects. 6.3 and 6.4.

\subsection{Finite disk correction and changes in ionization}

The combined effect of the finite disk correction factor and changes in ionization may be estimated by comparing results from the present calculations with the theoretical predictions from other authors, who used the improved version of the CAK theory, which takes both effects into account. To eliminate the uncertainties of the force multipliers from our calculations, the values according to Kudritzki (2002) are used for this purpose. The inclusion of the finite disk correction alone should lead to lower mass-loss rates and higher terminal velocities by factors of 1.5 to four (Lamers \& Cassinelli 1999).

Kudritzki (2002) calculated mass-loss rates for massive and luminous stars. Two examples of stellar parameters for the case $T_{\text {eff }}=50000 \mathrm{~K}$ are given in Table 4 .

Ideally, our results for these stellar parameters should agree with Kudritzki's ones. As the finite disk correction and changes in ionization have been neglected in the present calculations, however, the results will usually disagree. This can be seen from the comparison of both results in Fig. 11. In the last column of Table 4 the values of $n_{\mathrm{e}} / W$ obtained iteratively as described in 
Table 4. Stellar parameters and adopted values of $n_{\mathrm{e}} / W$ for the comparison with the mass-loss predictions from Kudritzki (2002), with $T_{\text {eff }}=50000 \mathrm{~K}$ in all cases.

\begin{tabular}{cccclc}
\hline \hline $\log L / L_{\odot}$ & $\begin{array}{c}\log g \\
(\mathrm{cgs})\end{array}$ & $\begin{array}{c}R_{*} \\
{\left[R_{\odot}\right]}\end{array}$ & $\begin{array}{c}M_{*} \\
{\left[M_{\odot}\right]}\end{array}$ & $\begin{array}{l}Z / Z_{\odot} \\
{\left[Z_{\odot}\right]}\end{array}$ & $\begin{array}{c}\log n_{\mathrm{e}} / W \\
{\left[\mathrm{~cm}^{-3}\right]}\end{array}$ \\
\hline 7.03 & 3.63 & 43.76 & 298 & 1.0 & 14.11 \\
& & & & 0.2 & 14.15 \\
& & & & 0.01 & 13.42 \\
& & & & 0.001 & 12.69 \\
6.42 & \multirow{3}{*}{3.85} & \multirow{2}{*}{21.71} & \multirow{2}{*}{122} & 0.0001 & 11.89 \\
& & & & 0.2 & 13.54 \\
& & & & 0.01 & 13.36 \\
& & & & 0.001 & 11.64 \\
\hline
\end{tabular}
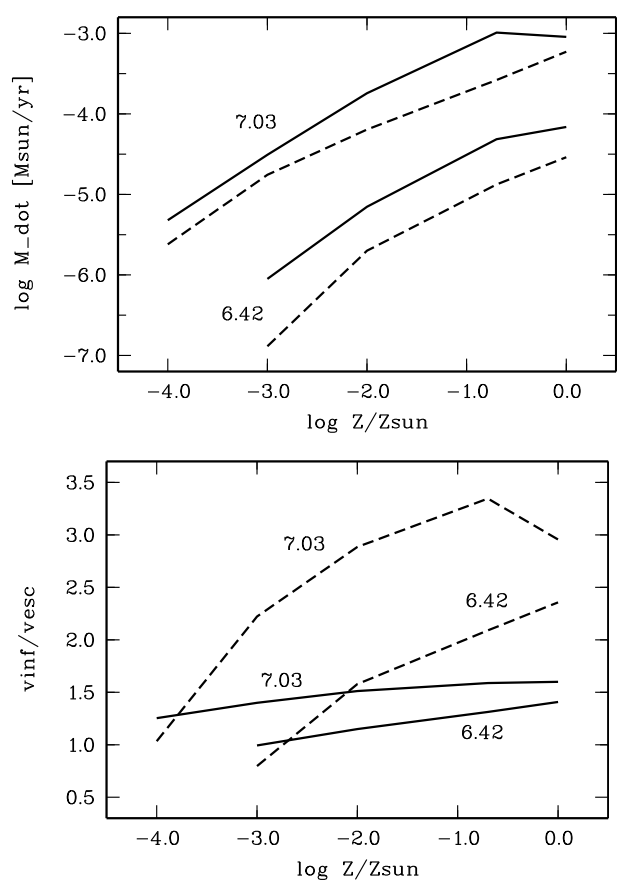

Fig. 11. Comparison of the predicted mass-loss rates (top) and terminal velocities (bottom) as a function of metallicity according to the present computational method (solid lines) with the results from Kudritzki (2002, dashed lines) for $\log L / L_{\odot}=7.03$ and 6.42, respectively, with $T_{\text {eff }}=50000 \mathrm{~K}$. In the present calculations, Kudritzki's force multipliers have been used as described in Sect. 2.4.1.

Sect. 2.4.1 are tabulated. In the present calculations these values are assumed to be constant throughout the wind.

From the comparison it can be seen that our mass-loss rates in all cases are higher than Kudritzki's. In the various cases the discrepancies vary by a factor of 1.5 up to a factor of seven. The terminal velocities are lower than Kudritzki's in most cases. Only for extremely low metallicities, the predicted terminal velocities approximately agree.

These results correspond to the expectations. The effect of ionization may be more or less strong, depending on which elements and which ionization states primarily contribute to the radiative force. In some cases the combined effect of finite disk correction and ionization may lead to greater discrepancies than would be expected from the finite disk correction alone. In other cases, both simplifications may almost compensate each other, so that the results agree approximately with results from the improved version of the CAK theory.
Table 5. Comparison of the mass-loss predictions for $Z / Z_{\odot}=1$ with the results from Pauldrach et al. (2004) in the upper part of the table and Pauldrach et al. (1988) in the lower. For each set of stellar parameters the results from Pauldrach et al. are given in the lower line.

\begin{tabular}{ccccrc}
\hline \hline $\begin{array}{c}T_{\text {eff }} \\
{[K]}\end{array}$ & $\begin{array}{c}\log g \\
(\mathrm{cgs})\end{array}$ & $\begin{array}{c}M_{*} \\
{\left[M_{\odot}\right]}\end{array}$ & $\begin{array}{c}\log \dot{M} \\
{\left[M_{\odot} / \mathrm{yr}\right]}\end{array}$ & $\begin{array}{r}v_{\infty} \\
{\left[\mathrm{km} \mathrm{s}^{-1}\right]}\end{array}$ & $\begin{array}{c}\log n_{\mathrm{e}} / \mathrm{W} \\
{\left[\mathrm{cm}^{-3}\right]}\end{array}$ \\
\hline 40000 & 3.70 & 0.410 & -7.28 & 296 & 12.9 \\
& & & -7.74 & 420 & \\
40000 & \multirow{2}{*}{3.80} & \multirow{2}{*}{1.110} & -7.10 & 431 & 12.7 \\
& & & -7.21 & 850 & \\
40000 & \multirow{2}{*}{3.35} & \multirow{2}{*}{1.000} & -5.73 & 194 & 13.8 \\
& & & -5.82 & 400 & \\
40000 & \multirow{2}{*}{3.69} & \multirow{2}{*}{0.644} & -7.06 & 332 & 12.9 \\
& & & -7.42 & 800 & \\
40000 & \multirow{2}{*}{4.45} & \multirow{2}{*}{0.546} & -8.78 & 580 & 12.0 \\
& & & -8.85 & 1881 & \\
50000 & \multirow{2}{*}{3.74} & \multirow{2}{*}{1.000} & -5.95 & 302 & 13.9 \\
& & & -5.88 & 500 & \\
50000 & \multirow{2}{*}{4.08} & \multirow{2}{*}{0.644} & -6.90 & 487 & 13.4 \\
& & & -7.34 & 910 & \multirow{2}{*}{50000} \\
\multirow{2}{*}{4.91} & \multirow{2}{*}{0.546} & -8.55 & 812 & 12.6 \\
& & & -9.00 & 2583 & \\
\hline
\end{tabular}

In Table 5 mass-loss rates and terminal velocities obtained with the present computational method and force multipliers according to Kudritzki (2002) are compared with predictions from Pauldrach et al. (2004) and Pauldrach et al. (1988) for central stars of planetary nebulae with a solar composition. The adopted values of $n_{\mathrm{e}} / W$ are given in the last column.

In one case only are the mass-loss rates from our calculations slightly lower (by a factor of 1.2) than the ones of Pauldrach et al. (1988). In all other cases, they are higher up to a factor of three. The terminal velocities in all cases are lower than the ones from Pauldrach et al. by factors between about 1.5 and three. This again agrees with the expected tendency of the predicted mass-loss rates of the present paper to be too large, whereas the terminal velocities are too low.

\subsection{The effect of line shadowing}

That the major contribution to the radiative force is due to a few strong lines for small wind optical depth parameters (see Sect. 5.1) has important consequences. In the present calculations the emergent monochromatic flux $F_{v}$ at the frequency of a line, from which the force multiplier is obtained (see Eq. (19)), corresponds to the continuum flux. In Fig. 12, for the line CIII 2977.0 $\AA$ and for the case $T_{\text {eff }}=30000 \mathrm{~K}, \log g=5.5$, and solar metal abundances, this flux is compared to the emergent flux from a LTE model atmosphere for sdB stars as used for spectral analyses (Heber, priv. comm.). It can be seen that near the line centre this flux is lower by about a factor of 100 than the flux that has been assumed in the mass-loss calculations. Thus in the inner parts of the wind where the velocity and thus the Doppler shift is low, $F_{v}$ has clearly been overestimated. For larger Doppler shifts, both fluxes approach each other and approximately agree for $\Delta \lambda \gtrsim 3.5 \AA$. These Doppler shifts correspond to velocities $v \gtrsim 1000 \mathrm{~km} \mathrm{~s}^{-1}$, which may be compared with the surface escape velocity $v_{\text {esc }}=955 \mathrm{~km} \mathrm{~s}^{-1}$ for this example with $\log g=5.5$. Thus the flux assumed in the calculations is approximately correct for the outer parts of the wind where $v \gtrsim v_{\text {esc }}$; however, it is clearly overestimated in the inner parts.

According to the results of Babel (1996) this effect of "line shadowing" should lead to lower mass-loss rates and higher 


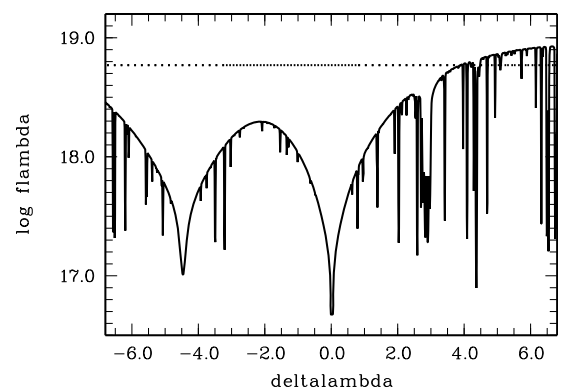

Fig. 12. Physical flux (in erg $\mathrm{cm}^{-3} \mathrm{~s}^{-1}$ ) near the line CIII $\lambda 977.0 \AA$ for $T_{\text {eff }}=30000 \mathrm{~K}, \log g=5.5$ and solar metal abundances as a function of $\Delta \lambda=\lambda-977.0 \AA$. The solid line represents the flux from a model atmosphere (Heber, priv. comm.), the dotted line represents the flux used in the present calculations.

terminal velocities in comparison to the usual theory that assumes a velocity-independent flux. Then terminal velocities of the order $v_{\infty} \approx 5 v_{\text {esc }}$ and mass-loss rates that are lower by a factor of the order ten are not unusual. Thus we expect that the present calculations overestimate the mass-loss rates. The agreement with the results of Vink \& Cassisi (2002) does not contradict this, because the terminal velocity is a free parameter in their calculations. If a terminal velocity $v_{\infty}=5 v_{\text {esc }}$ instead of $v=v_{\text {esc }}$ were assumed, then it follows from the dependence of $\dot{M}$ on the assumed value of $v_{\infty}$ derived in Vink et al. (2000) that the massloss rates may indeed be lower by a factor around ten. The consequence of lower mass-loss rates would be that decoupling of the metals occurs at lower gravities than predicted according to the present results.

Qualitatively, the inclusion of the effect of line shadowing should have similar consequences to including the finite disk correction (lower mass-loss rates, higher terminal velocities). Quantitatively, however, it seems to be much stronger for the weak winds discussed in the present paper. According to Owocki \& ud-Doula (2004) the finite disk correction reduces the radiative force in the inner parts of the wind only by a factor of about two, whereas the example shown in Fig. 12 shows that line shadowing may reduce the radiative force due to a strong line up to two orders of magnitude.

The results of Babel (1996) predict increasing terminal velocities for increasing surface gravities and otherwise fixed stellar parameters, whereas the present results shown in Sect. 4 predict a decreasing tendency for $v_{\infty}$. The effect of line shadowing probably also changes the dependencies of the mass-loss rates and of the terminal velocities on the metallicity.

\subsection{Consequences for the chemical composition}

The results presented in Sects. 4 and 5 have shown that, in hot white dwarfs and in the majority of sdB stars (see Sect. 7), no chemically homogeneous winds can exist, at least if the metallicity is not higher than solar. Thus, if any mass-loss exists, it should change the surface composition. The only exception may be that the decoupling of the metals from the passive plasma occurs at a radius, at which the velocity of hydrogen and helium already exceeds the local escape velocity. Then hydrogen and helium can still be expelled from the star. For the case where decoupling occurs before the local escape velocity has been reached, Porter \& Skouza (1999) propose a periodic timedependent scenario. The passive plasma decelerates after decoupling and eventually stalls. Thus a shell of gas is generated that reaccretes to the star. In the course of time, this scenario should lead to a depletion of metals near the stellar surface.

Another possible scenario is a pure metallic wind with hydrostatic hydrogen and helium. For main sequence A stars, this has been discussed by Babel (1995). If the stellar atmosphere is approximately in a stationary state, then the metals must have an outward movement. This leads to an outward frictional force on hydrogen and helium. If these elements are in hydrostatic equilibrium $\left(v_{1}=0\right)$ and if we demand that their partial pressure decreases in an outward direction $\left(\mathrm{d} p_{1} / \mathrm{d} r<0\right)$, then it follows from the momentum Eq. (24) for hydrogen and helium that the outward frictional acceleration must be less than the gravitational one:

$g_{\text {coll }}^{(1)}<\frac{G M_{*}}{r^{2}}$.

Otherwise the outward frictional force on the passive plasma due to the outflowing metals would be so strong that it cannot be in hydrostatic equilibrium. As the density in the stellar atmosphere is much higher than in the wind, it is a reasonable assumption that the drift velocities of the metals are small in comparison to the thermal velocity. Then the collisional acceleration on hydrogen and helium can be calculated from the linear approximation. For $x \ll 1$ it is $G(x) \approx \frac{2}{3} \frac{1}{\sqrt{\pi}} x$, so that the momentum exchanged per unit volume and unit time via Coulomb collisions may be written as

$|\Delta Q|=n_{1} n_{2} l_{12}\left|v_{2}-v_{1}\right|$

with

$l_{12}=\frac{16}{3} \sqrt{\pi} \frac{Z_{1}^{2} Z_{2}^{2} e^{4}}{m_{12} \alpha^{3}} \ln \Lambda$.

The quantity $n_{1} n_{2} l_{12}$ corresponds to the resistance coefficient $K_{\text {st }}$ derived by Burgers (1969; see his Eq. (24.14)). With Eqs. (29), (52), (53), with $v_{1}=0$, and the equation of continuity for the mean metal, it follows for the collisional acceleration on hydrogen and helium that

$g_{\text {coll }}^{(1)}=\frac{1}{m_{1} m_{2}} \frac{\dot{M}_{2}}{4 \pi r^{2}} l_{12}$.

Then condition (51) can be written as

$\dot{M}_{2}<4 \pi G M_{*} \frac{m_{1} m_{2}}{l_{12}}$.

If the mass-loss rate of the metals fulfils this condition, then the collisional acceleration on the passive plasma never can exceed gravity, independent of how high the velocity of the mean metal is (because the linear approximation leads to an upper limit of $g_{\text {coll }}^{(1)}$ ). With $M_{*}=0.5 M_{\odot}$, mean charges $Z_{1}=1.0, Z_{2}=3.0$, mean masses $m_{1}=1.3 m_{\mathrm{p}}, m_{2}=14.6 m_{\mathrm{p}}$, and with $\ln \Lambda=12.0$, it follows for a temperature $T=35000 \mathrm{~K}$ that

$\dot{M}_{2}<3 \times 10^{-16} \frac{M_{\odot}}{\mathrm{yr}}$

Thus hydrogen and helium may be in hydrostatic equilibrium if the mass-loss rate of the metals is lower than about $10^{-16} M_{\odot} / y$. On the other hand, we know from the previous results that the existence of a coupled wind requires a total mass-loss rate greater than at least $10^{-12} M_{\odot} / y$. For an approximately solar mass fraction of the metals of the order of $10^{-2}$, this means that a coupled wind requires a mass-loss rate of the metals alone $\dot{M}_{2}>10^{-14} M_{\odot} /$ yr. If, however, $\dot{M}_{2}$ were somewhere in between 
these limits, e.g. $\dot{M}_{2} \approx 10^{-15} M_{\odot} / \mathrm{yr}$, then neither of the two cases seems to be possible. This value is too low for the existence of a coupled wind and too high for the case with hydrostatic passive plasma. Then hydrogen and helium may either fall back onto the star or be expelled, depending on at which radius decoupling occurs. Which of the various scenarios is the appropriate one essentially depends on the flow of the metals. In the following we discuss the case with hydrostatic hydrogen and helium and with a pure metallic wind. If the metals are trace elements and the densities in the wind are sufficiently low, then the mass-loss rates of the various metals should be independent of each other.

If the mass-loss rate of a metal is greater than zero and if the atmosphere is approximately in a stationary state, the metal must have an outward velocity. If the effect of concentration gradients is weak, then the radiative force must not only balance gravitational settling, but also must compensate the inward frictional force on the metal due to collisions with the passive plasma. Due to saturation effects, the radiative force on a metal increases with decreasing abundance. Consequently, the abundances of these metals with non-zero mass-loss rates tend to be lower than the abundances obtained from diffusion calculations that assume an equilibrium between the downward gravitational force and the upward radiative force. Only for these metals, for which the mass-loss rate is sufficiently low, should the equilibrium abundances agree with the ones derived from spectral analyses.

Seaton $(1996,1999)$ presented the results of time-dependent diffusion calculations for iron group elements in the stellar envelopes of HgMn stars and allowed for an outflow of these elements at the outer boundary. The abundances are predicted to vary with time. It may in principle happen that two stars with similar stellar parameters and surface compositions have different internal compositions. In the absence of strong concentration gradients, a maximum possible value for the outward flow of a metal can be derived for each depth, if the radiative acceleration is known as a function of the abundance. This maximum of the flow is small in regions where the metal has a noble gas configuration and thus the radiative acceleration is low. In Seaton (1999), these regions are referred to as barriers. How the surface abundance of a metal changes with time should depend essentially on its mass-loss rate and on the location of these barriers in the stellar envelope.

The abundance patterns of the various types of chemically peculiar stars discussed in the present paper qualitatively agree with these expectations, although exceptions exist. For sdB stars, equilibrium abundances have been predicted and compared with measured ones e.g. by Bergeron et al. (1988); Charpinet et al. (1997); Ohl et al. (2000), and Behara \& Jeffery (2007). For some elements (e.g. Fe and $\mathrm{N}$ ) there is generally good agreement, whereas for others (e.g. Si) the predicted values may be too high by several orders of magnitude. According to Chayer et al. (2006), the measured abundances of $\mathrm{Ge}, \mathrm{Zr}$, and $\mathrm{Pb}$ are somewhere between the solar value and the predicted equilibrium abundances, which for these elements exceed the solar value by about two orders of magnitude. The wide spread of the measured abundances may be an indication of a time-dependent scenario.

For hot hydrogen-rich white dwarfs, metal abundances have been measured or predicted e.g. by Barstow et al. (2003, 2005), Chayer et al. (1995a,b); Good et al. (2005); Schuh et al. (2005); Dobbie et al. (2005), and Vennes et al. (2005, 2006). For some elements ( $\mathrm{Fe}$ and $\mathrm{O})$, the agreement is close in many cases, for others (e.g. C, N, and $\mathrm{Ni}$ ) the abundances predicted from equilibrium diffusion theory tend to be too large. An exception is silicon, for which the measured abundances are usually larger than the predicted ones. Thus in hot hydrogen-rich white dwarfs and in some helium-rich ones (Dreizler 1999) the situation is in some respect similar to sdB stars. The predicted abundances tend to be larger than the measured ones.

Another example is found in the HgMn stars. The manganese abundances in the stars analysed by Jomaron et al. (1999) in all cases are lower than predicted from the equilibrium diffusion calculations of Alecian \& Michaud (1981) and greater than the solar values. Nitrogen is typically depleted by at least a factor of 100 , which is more than predicted from diffusion theory (Roby et al. 1999). The abundances of neon scatter from slight deficiencies to underabundances by one order of magnitude or even more (Dworetsky \& Budaj 2000). This may point to some timedependent process. The results of Seaton $(1996,1999)$ for various iron group elements show that the presence of an outflow at the outer boundary in general leads to better agreement. An exception to the expected tendency is mercury, for which abundances greater than predicted from equilibrium diffusion theory have been detected (Proffitt et al. 1999). However, at least in some HgMn stars, elements seem to be distributed inhomogeneously over the surface (Hubrig et al. 2006), which complicates the problem.

\subsection{Selective winds or turbulence?}

As explained in the preceeding section, in the presence of pure metallic winds the abundances of the metals tend to be lower than predicted from equilibrium diffusion calculations. This should be so for overabundant, as well as for deficient, metals. An alternative explanation of the discrepancies between measured and predicted abundances may be the presence of turbulence, which could stem from mixing processes like convection or stellar rotation. Then, however, the abundances of the deficient metals should be larger than predicted from equilibrium diffusion calculations (Vauclair et al. 1978), because turbulence tends to level out concentration gradients and reduces the effect of gravitational settling. Additional mixing due to turbulence outside of convection zones has been assumed by Richer et al. (2000) in their calculations for AmFm stars (which represent the continuation of the HgMn phenomenon at lower effective temperatures) to reduce the amplitude of the predicted abundance anomalies to a level that agrees with observational results.

In hot hydrogen-rich white dwarfs and sdB stars, however, the abundances not only of enriched metals are lower than predicted, but also those of deficient metals. In particular, the effect of turbulence alone can hardly explain why elements that should be overabundant according to diffusion theory are in fact deficient, as has been found for argon in two of the hottest known DA white dwarfs (Werner et al. 2007). Thus the scenario with selective winds seems to be the most likely one for these stars.

The only element that is generally less deficient than predicted from equilibrium diffusion calculations, is helium. This is so for these hot white dwarfs, in which helium is detectable (Vennes et al. 1988), sdB (Michaud et al. 1989), and HgMn stars (Michaud et al. 1979). According to the results of Krtička (2006) for peculiar B stars, this can hardly be explained within the framework of selective winds. Thus the helium deficiencies should be due to gravitational settling. It is possible that completely undisturbed stellar atmospheres hardly exist and some turbulence is always present. This may explain that helium sinks more slowly than expected. However, it is still unclear what the origin of this turbulence is exactly. 


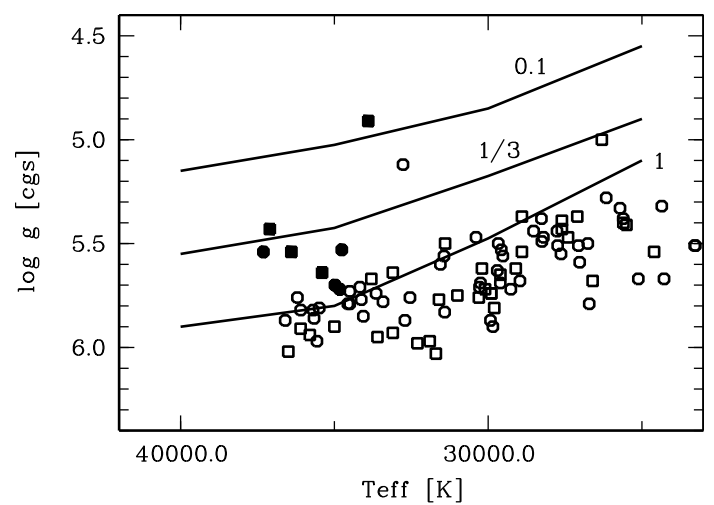

Fig. 13. Lines in the $T_{\text {eff }}-\log g$ diagram above which chemically homogeneous winds may exist for $Z / Z_{\odot}=0.1,1 / 3$ and 1 , respectively. Squares and circles represent the sdB stars analysed by Maxted et al. (2001) and Lisker et al. (2005), respectively. Filled symbols represent the sdB's with peculiar $\mathrm{H}_{\alpha}$ line profiles, which may indicate the presence of a weak wind.

\section{Summary and conclusions}

In the $T_{\text {eff }}-\log g$ diagram of Fig. 13 for $25000 \mathrm{~K} \leq T_{\text {eff }} \leq$ $40000 \mathrm{~K}$ and for $Z / Z_{\odot}=1,1 / 3$ and 0.1 , the lines are shown above which chemically homogeneous winds may exist according to the present results. The samples of sdB stars analysed by Maxted et al. (2001) and Lisker et al. (2005) shown in the figure have been analysed for peculiar $\mathrm{H}_{\alpha}$ line profiles, which may be interpreted as wind signatures according to Heber et al. (2003) and Vink (2004). As can be seen in the figure, such signatures have only been detected in the most luminous sdB stars. According to the calculations of stellar evolution by Dorman et al. (1993), these ones are in a post-EHB stage of evolution.

In the figure it can be seen that the majority of $\mathrm{sdB}$ stars populate a region in the $T_{\text {eff }}-\log g$ diagram that is just below the line above which for $Z / Z_{\odot}=1$ coupled winds may exist. If multicomponent effects are neglected, the results predict weak winds with mass-loss rates $\dot{M} \lesssim 10^{-12} M_{\odot} /$ yr. However, for such weak winds the momentum exchange between the metals, on the one hand, and hydrogen and helium, on the other, is not effective enough, because the densities in the wind are too low. Possible winds should be selective winds that lead to additional changes in the surface composition, which have not yet been taken into account in the diffusion calculations with and without mass-loss.

According to the present results coupled winds can exist for the most luminous sdB stars, if the metallicity is not too low. This would explain why only in these stars wind signatures have been detected. The predicted mass-loss rates are of the order of $10^{-11}$ to $10^{-10} M_{\odot} / \mathrm{yr}$. This agrees with the predictions of the mass-loss recipe of Vink \& Cassisi (2002), nevertheless the existence of these winds is still uncertain from the theoretical point of view. In Vink \& Cassisi's calculations, the terminal velocity has been a free parameter, and their results are for $v_{\infty}=v_{\text {esc }}$. The assumption of higher terminal velocities would lead to lower mass-loss rates. In the present calculations, the finite disk correction and the shadowing of the flux by the photospheric lines have not been taken into account. Especially the latter effect seems to be very important. As discussed in Sect. 6, both simplifications should lead to the mass-loss rates being overestimated and the terminal velocities being underestimated. Then for a star with given stellar parameters and chemical composition, the densities in the wind would be lower. Consequently decoupling of the metals from hydrogen and helium would occur at lower gravities than predicted, and the lines shown in Fig. 13 would be shifted to lower gravities. Frictional heating may have a similar effect. In addition, the presence of density inhomogenities in the wind, which are not taken into account in the present calculations, may possibly change the mass-loss rates. This effect of "clumping" is still investigated (Oskinova et al. 2007). For luminous stars there is some evidence that the mass-loss rates may need to be revised downwards (e.g. Bouret et al. 2005; Fullerton et al. 2006; Martins et al. 2005; Puls et al. 2006).

To confirm the existence of coupled winds in the most luminous sdB stars, more sophisticated calculations are necessary. Because the sdB stars have a variety of abundances, it is important to show which elements contribute primarily to the radiative force. As explained by Puls et al. (2000), this should be the most abundant elements with the strongest lines in weak winds. According to the present results, the most important element is carbon for $T_{\text {eff }}=25000$ and $30000 \mathrm{~K}$. For higher effective temperatures the situation is less clear.

If during the post-EHB evolution indeed winds with massloss rates $\dot{M} \gtrsim 10^{-11} M_{\odot} /$ yr were initiated, the outer layers of the star will be removed rapidly. This should lead to the abundance anomalies gradually decreasing, so elements that are not detectable in sdB stars within the EHB band may reappear on the surface during the post-EHB evolution. This may explain the result of Edelmann et al. (2006) that, in those sdB stars with $T_{\text {eff }} \gtrsim 32000 \mathrm{~K}$ near the EHB band, no Si, Mg, and Al have been detected, whereas these elements are present in their more luminous counterparts with similar effective temperatures, which are in a post-EHB stage of evolution.

The results confirm the present picture of white-dwarf chemical evolution. The variety of compositions from hydrogen-rich to helium-carbon-oxygen-rich observed in pre-white dwarfs is due to the dredge up of processed material (Werner \& Herwig 2006). Mass-loss rates between about $10^{-11}$ and $10^{-6} M_{\odot} / \mathrm{yr}$ are predicted for pre-white dwarfs with $M_{*}=0.5 M_{\odot}, 30000 \mathrm{~K} \leq$ $T_{\text {eff }} \leq 50000 \mathrm{~K}$, and $3.4 \lesssim \log g \lesssim 5.0$, dependent on stellar parameters and metallicities. These mass-loss rates are large enough to prevent diffusion, at least if the metallicity is not reduced by more than about a factor of ten in comparison to the solar value. No wind solution exists for white dwarfs on the cooling sequence with similar effective temperatures. Even for solar metallicity, the maximum possible radiative acceleration is too low by about one order of magnitude. Thus somewhere during the evolution with $T_{\text {eff }}>50000 \mathrm{~K}$, the mass-loss rates must decrease to very low values so that chemically homogeneous winds can no longer exist. The possibility that metallic winds still exist may explain the result of e.g. Good et al. (2005) that neither diffusion calculations that assume chemically homogeneous winds nor diffusion calculations that assume the absence of any massloss can explain the measured abundances in DA and DAO white dwarfs very well.

As discussed in Sect. 6.3, the existence of pure metallic winds with mass-loss rates $\dot{M} \lesssim 10^{-16} M_{\odot} / \mathrm{yr}$ and with hydrostatic hydrogen and helium may be a promising scenario to explain the abundance anomalies of the metals in the various types of chemically peculiar stars considered in this paper. Then, however, the problem remains, why the abundances of helium in general are larger than predicted from diffusion calculations, which assume an equilibrium between gravitational settling and radiative levitation. Possibly the stellar atmospheres are not completely undisturbed after all.

Hot (pre-) white dwarfs already have a variety of helium (and metal-) abundances before the onset of gravitational settling is expected. A dependence on the star's history may also play some role in sdB stars. According to the analyses of 
Edelmann et al. (2003) and Lisker et al. (2005) two distinct sequences of sdB stars seem to exist, which are characterised by an offset in the helium abundances. This phenomenon can hardly be explained with a single atmospheric effect.

Acknowledgements. I would like to thank J. Vink for doing some additional mass-loss calculations, U. Heber for the calculation of a synthetic spectrum, A. Feldmeier for useful hints concerning the CAK theory, and I. Bues for carefully reading the manuscript. The referee's comments have been a great help in improving the paper significantly.

\section{References}

Abbott, D. C. 1982, ApJ, 259, 282

Adelman, S. J., Caliskan, H., Gulliver, A. F., \& Teker, A. 2006, A\&A, 447, 685

Alecian, G., \& Michaud, G. 1981, ApJ, 245, 226

Babel, J. 1995, A\&A, 301, 823

Babel, J. 1996, A\&A, 309, 867

Barstow, M. A., Good, S. A., Holberg, J. B., et al. 2003, MNRAS, 341, 870

Barstow, M. A., Cruddace, R. G., Kowalski, M. P., et al. 2005, MNRAS, 362, 1273

Behara, N., \& Jeffery, S., Proc. of the 15th European workshop on White Dwarfs, in press

Bergeron, P., Wesemael, F., Michaud, G., \& Fontaine, G. 1988, ApJ, 332, 964 Bjorkman, J. 1995, ApJ, 453, 369

Blanchette, J. P., Chayer, P., Wesemael, F., et al. 2006, Baltic Astronomy 15, 301

Bouret, J.-C., Lanz, T., \& Hillier, D. J. 2005, A\&A, 438, 301

Burgers, J. M. 1969, Flow equations for composite gases (New York: Academic Press), 1

Castor, J. I., Abbott, D. C., \& Klein, R. I. 1975, ApJ, 195, 157 (CAK)

Charpinet, S., Fontaine, G., Brassard, P., et al. 1997, ApJ, 483, L123

Charpinet, S., Fontaine, G., Brassard, P., Chayer, P., \& Green, E. M. 2006, Baltic Astron., 15, 305

Chayer, P., Fontaine, G., \& Wesemael, F. 1995a, ApJS, 99, 189

Chayer, P., Vennes, S., Pradhan, K. A., et al. 1995b, ApJ, 454, 429

Chayer, P., Fontaine, G., Fontaine, M., et al. 2004, Ap\&SS, 291, 379

Chayer, P., Fontaine, F., Fontaine, G., Wesemael, F., \& Dupuis, J. 2006, Baltic Astron., 15, 131

Dobbie, P. D., Barstow, M. A., Hubeny, I., et al. 2005, MNRAS, 363, 763

Dorman, B., Rood, R. T., \& O’Connell, R. W. 1993, ApJ, 419, 596

Dreizler, S. 1999, A\&A, 352, 632

Dreizler, S., \& Wolff, B. 1999, A\&A, 348, 189

Dworetsky, M. M., \& Budaj, J. 2000, MNRAS, 318, 1264

Edelmann, H., Heber, U., Hagen, H. J., et al. 2003, A\&A, 400, 939

Edelmann, H., Heber, U., \& Napiwotzki, R. 2006, Baltic Astronomy, 15, 103

Feldmeier, A., \& Nikutta, R. 2006, A\&A, 446, 661

Feldmeier, A., \& Shlosman, I. 2002, ApJ, 564, 385

Fontaine, G., \& Chayer, P. 1997, The Third Conference on Faint Blue Stars, ed.

A. G .D. Philip, J. Liebert, \& R. A. Saffer (Davis Press), 169

Fontaine, G., Brassard, P., Charpinet, S., et al. 2003, ApJ, 597, 518

Fontaine, G., Green, E. M., Chayer, P., et al. 2006, Baltic Astron., 15, 211

Friend, D., \& Abbott, D. C. 1986, ApJ, 311, 701

Fullerton, A. W., Massa, D. L., \& Prinja, R. K. 2006, ApJ, 637, 1025

Gayley, K. 2000, ApJ, 529, 1019

Geier, S., Heber, U., \& Napiwotzki, R. 2007, A\&A, in preparation

Good, S. A., Barstow, M. A., Burleigh, M. R., et al. 2005, MNRAS, 363, 183

Grevesse, N., \& Sauval, A. J. 1998, SSRv, 85, 161

Groth, H. G., Kudritzki, R. P., \& Heber, U. 1985, A\&A, 152, 107

Hamann, W. R., Gruschinske, J., Kudritzki, R. P., \& Simon, K. P. 1981, A\&A, 104, 249

Heber, U. 1986, A\&A, 155, 33

Heber, U., \& Edelmann, H. 2004, Ap\&SS, 291, 341

Heber, U., Napiwotzki, R., \& Reid, J. N. 1997, A\&A, 323, 819

Heber, U., Reid, I. N., \& Werner, K. 2000, A\&A, 363, 198

Heber, U., Maxted, P. F. L., Marsh, T. R., Knigge, C., \& Drew, J. E. 2003, in Stellar Atmosphere Modeling, ed. I. Hubeny, D. Mihalas, K. Werner, ASP Conf. Ser., 288, 251

Herald, J. E., Bianchi, L., \& Hillier, D. J. 2005, ApJ, 627, 424

Hubrig, S., Gonzáles, J. F., Savanov, I., et al. 2006, MNRAS, 371, 1953

Jomaron, C. M., Dworetsky, M. M., \& Allen, C. 1999, MNRAS, 303, 555

Jordan, S., Werner, K., \& O'Toole, S. J. 2005, A\&A, 432, 273

Jordan, S., Aznar Cuadrado R., Napiwotzki R., Schmid H. M., \& Solanki S. K. 2007, A\&A, 462, 1097
Karl, C. A., Napiwotzki, R., Heber, U., et al. 2005, A\&A, 434, 637

Koester, D., Dreizler, S., Weidemann, V., \& Allard, N. F. 1998, A\&A, 338, 612 Koesterke, J., \& Werner, K. 1998, ApJ, 500, L55

Koesterke, L., Dreizler, S., \& Rauch, T. 1998, A\&A, 330, 1041

Krtička, J. 2006, MNRAS, 367, 1282

Krtička, J., \& Kubát, J. 2000, A\&A, 359, 983

Krtička, J., \& Kubát, J. 2001a, A\&A, 369, 222

Krtička, J., \& Kubát, J. 2001b, A\&A, 377, 175

Krtička, J., \& Kubát, J. 2002, A\&A, 388, 531

Krtička, J., Owocki, S. P., Kubát, J., Galloway, R. K., \& Brown, J. C. 2003, A\&A, 402, 713

Krtička, J., Kubát, J., \& Groote, D. 2006, A\&A 460, 165

Kudritzki, R. P. 2002, ApJ, 577, 389

Kudritzki, R. P., \& Puls, J. 2000, ARA\&A, 38, 613

Kudritzki, R. P., Urbaneja, M. A., \& Puls, J. 2006, IAU Symp., 234, 119

Lamers, H. J. G. L. M., \& Cassinelli, J. P. 1999, Introduction to stellar winds (Cambbridge Univ. Press)

Landstreet, J. D., Dolez, N., \& Vauclair, S. 1998, A\&A, 333, 977

Lisker, T., Heber, U., Napiwotzki, R., et al. 2005, A\&A, 430, 223

Lucy, L. B. 2007a, A\&A, 468, 649

Lucy L. B. 2007b, A\&A, 474, 701

Martins, F., Schaere, D., Hillier, D. J., et al. 2005, A\&A, 441, 735

Maxted, P. F. L., Heber, U., Marsh, T. R., \& North, R. C. 2001, MNRAS, 326, 1391

Michaud, G., Montmerle, T., Cox, A. N., et al. 1979, ApJ, 234, 206

Michaud, G., Bergeron, P., Heber, U., \& Wesemael, F. 1989, ApJ, 338, 417

Ohl, R. G., Chayer, P., \& Moos, H. W. 2000, ApJ, 538, L95

Oskinova, L. M., Hamann, W. R., \& Feldmeier, A. 2007, A\&A, 476, 1331

O'Toole, S. J., \& Heber, U. 2006, A\&A, 452, 579

O’Toole, S. J., Jordan, S., Friedrich, S., \& Heber, U. 2005, A\&A, 437, 227

Owocki, S. P., \& Puls, J. 2002, ApJ, 568, 965

Owocki, S. P., \& ud-Doula, A. 2004, ApJ, 600, 1004

Pauldrach, A W. A., Hofmann, T. L., \& Méndez, R. H. 2004, A\&A, 419, 1111

Pauldrach, A., Puls, J., \& Kudritzki, R. P. 1986, A\&A, 164, 86

Pauldrach, A., Puls, J., Kudritzki, R. P., \& Méndez, R. H. 1988, A\&A, 207, 123

Porter, J. M., \& Skouza, B. A. 1999, A\&A, 344, 205

Press, W. H., Teukolsky, S. A., Vetterling, W. T., \& Flannery, B. R. 1992,

Numerical Recipes (Cambridge University Press)

Proffitt, C. R., Brage, T., Leckrone, D., et al. 1999, ApJ, 512, 942

Puls, J., Springmann, U., \& Lennon, M. 2000, A\&AS, 141, 23

Puls, J., Markova, N., Scuderi, S., et al. 2006, A\&A, 454, 625

Rauch, T. 1993, A\&A, 276, 171

Richer, J., Michaud, G., \& Turcotte, S. 2000, ApJ, 529, 338

Roby, S. W., Leckrone, D. S., \& Adelman, S. J. 1999, ApJ, 524, 974

Saffer, R. A., Bergeron, P., Koester, D., \& Liebert, J. 1994, ApJ, 432, 351

Schuh, S. L., Dreizler, S., \& Wolff, B. 2002, A\&A, 382, 164

Schuh, S. L., Barstow, M. A., \& Dreizler, S. 2005, ASP Conf. Ser., 334, 237

Seaton, M. J. 1996, Ap\&SS, 237, 107

Seaton, M. J. 1999, MNRAS, 307, 1008

Smith, K. C. 1996, Ap\&SS, 237, 77

Springmann, U. W. E., \& Pauldrach, A. W. A. 1992, A\&A, 262, 515

Tinkler, C. M., \& Lamers, H. J. G. L. M. 2002, A\&A, 384, 987

Turcott, S. 2003, ASPC, 305, 199

Unglaub, K., \& Bues, I. 1996, A\&A, 306, 843

Unglaub, K., \& Bues, I. 1998, A\&A, 338, 75

Unglaub, K., \& Bues, I. 2000, A\&A, 359, 1042

Unglaub, K., \& Bue, I. 2001, A\&A, 374, 570

Vauclair, S. 2003, Ap\&SS, 284, 205

Vauclair, G., Vauclair, S., \& Michaud, G. 1978, ApJ, 223, 920

Vauclair G., Vauclair S., \& Greenstein J. L. 1979, A\&A, 80, 79

Vauclair, S., \& Vauclair, G. 1982, ARA\&A, 20, 37

Vennes, S., Pelletier, C., Fontaine, G., \& Wesemael, F. 1988, ApJ, 331, 876

Vennes, S., Chayer, P., \& Dupuis, J. 2005, ApJ, 622, L121

Vennes, S., Chayer, P., Dupuis, P., \& Lanz, T. 2006, ApJ, 652, 1554

Vink, J. S. 2004, in Extreme Horizontal Branch Stars and Related Objects, Ap\&SS, 291, 239

Vink, J. S., \& Cassisi, S. 2002, A\&A, 392, 553

Vink, J. S., de Koter, A., \& Lamers, H. J. G. L. M. 2000, A\&A, 362, 295

Votruba, J., Feldmeier, A., Kubát, J., \& Rätzel, D. 2007, A\&A, 474, 549

Werner, K., \& Herwig, F. 2006, PASPC, 118, 840

Werner, K., Rauch, T., \& Kruk, J. W. 2007, A\&A, 466, 317

Wiese, W. L., Smith, M. W., \& Glennon, B. M. 1966, Atomic Transition Probabilities, Nat. Bur. of Standards, Washington, D.C.

Zavala, R. T., Adelman, S. J., \& Hummel, C. A. 2007, ApJ, 655, 1046 